\title{
Discovery of Galactic O IV and O v X-ray absorption due to transition temperature gas in the PKS 2155-304 spectrum
}

\author{
J. Nevalainen ${ }^{1}$, B. Wakker ${ }^{2}$, J. Kaastra ${ }^{3,4}$, M. Bonamente ${ }^{5}$, S. Snowden ${ }^{6}$, F. Paerels ${ }^{7}$, and C. de Vries ${ }^{3}$ \\ 1 Tartu Observatory, Observatooriumi 1, 61602 Tõravere, Estonia \\ e-mail: jukka@to.ee \\ 2 Supported by NASA/NSF, affiliated with Department of Astronomy, University of Wisconsin-Madison, Madison, WI 53706, USA \\ 3 SRON, Sorbonnelaan 2, 3584 CA Utrecht, The Netherlands \\ ${ }_{5}^{4}$ Leiden Observatory, Leiden University, PO Box 9513, 2300 RA Leiden, The Netherlands \\ 5 University of Alabama in Huntsville, Huntsville, AL 35899, USA \\ 6 NASA/Goddard Space Flight Center, Greenbelt, MD 20771, USA \\ 7 Columbia University, 1022 Pupin, 550 West 120th Street, New York, NY 10027, USA
}

Received 22 December 2016 / Accepted 20 May 2017

\begin{abstract}
Far-ultraviolet (FUV) observations have revealed transition temperature gas (TTG; $\log T(\mathrm{~K}) \sim 5$ ), located in the lower Galactic halo and in high-velocity clouds. However, the corresponding X-ray absorption has so far remained mostly undetected. In order to make an improvement in this respect in Galactic X-ray absorption studies, we accumulated very deep ( $\sim 3 \mathrm{Ms})$ spectra of the blazar PKS 2155-304 obtained with the spectrometers RGS1, RGS2, LETG/HRC, and LETG/ACIS-S and studied the absorption lines due to the intervening Galactic components. The very high quality of the data and coverage of important wavelengths with at least two independent instruments allowed us to reliably detect $10 \mathrm{Galactic}$ lines with better than $99.75 \%$ confidence. We discovered significant absorption from blended O IV transitions $1 \mathrm{~s}-2 \mathrm{p}^{2} \mathrm{~S}(22.571 \AA), 1 \mathrm{~s}-2 \mathrm{p}{ }^{2} \mathrm{P}(22.741 \AA)$, and $1 \mathrm{~s}-2 \mathrm{p}{ }^{2} \mathrm{D}(22.777 \AA)$, and from the $\mathrm{O} v$ transition $1 \mathrm{~s}-2 \mathrm{p}(22.370 \AA)$ from TTG at $\log T(\mathrm{~K})=5.2 \pm 0.1$. A joint X-ray and FUV analysis indicated that photoionisation is negligible for this component and that the gas is in a cooling transition phase. However, the temperature is high enough that the column density ratio $N(\mathrm{O}$ IV $) / N(\mathrm{O} \mathrm{V})$ is not significantly different from that in collisional ionisation equilibrium (CIE). Under CIE we obtained $N_{\mathrm{OIV}}=3.6 \pm 2.0 \times 10^{15} \mathrm{~cm}^{-2}$, corresponding to $N_{\mathrm{H}}=1.0 \pm 0.5 \times 10^{19} \frac{Z_{\odot}}{Z_{\mathrm{TTG}}} \mathrm{cm}^{-2}$.
\end{abstract}

Key words. instabilities - line: identification - Galaxy: halo - X-rays: general

\section{Introduction}

The Galactic transition temperature gas (TTG; $\log T(\mathrm{~K}) \sim 5$ ) has been very robustly detected via far-ultraviolet (FUV) absorption observations of ions such as O VI, N v, C IV, and Si IV (e.g. Wakker et al. 2012). The kinematic structure of TTG is often complex because of the presence of the low- and high-velocity components.

The low-velocity TTG component that is FUV absorbing is likely located in the lower Galactic halo (see Wakker et al. 2012, for observations) and can be understood via the Galactic fountain model (e.g. Shapiro \& Field 1976). In this model the supernovae in the Galactic disk heat the interstellar medium to a temperature $\log T(\mathrm{~K}) \sim 6$, which consequently expands a few kpc away from the Galactic plane (e.g. Hagihara et al. 2010; Sakai et al. 2014), thus forming the hot phase of the Galactic halo. The cooling timescales are shorter than those required to reach a hydrostatic equilibrium and thus the cooled material falls towards the disk. The outcome of this process is a flow of material and regions containing TTG located in the lower Galactic halo.

Transition temperature gas at high velocities is likely more distant. Some of these are low-metallicity accreting clouds, others are parts of the Magellanic stream, while still others are suspected of being outside the Milky Way's halo. Transition temperature gas is expected to form around the cooler, denser clouds seen in 21-cm emission as they interact with the hot coronal gas.

In X-rays, the observational view on the Milky Way is less complete. The hot phase $(\log T(\mathrm{~K}) \sim 6)$ of the Galactic halo has been detected via soft X-ray emission (e.g. Snowden et al. 1991; Burrows \& Mendenhall 1991) and via O VII and O VIII absorption (e.g. Fang et al. 2015). However, owing to the relatively low efficiency and resolution of the high-resolution X-ray spectrometers to date, the X-ray features of the FUV-detected transition temperature gas have not yet been previously detected.

Our aim in this work is to improve our insight into Galactic hot gas physics using soft X-ray spectroscopy by analysing very deep co-added exposures of the bright blazar PKS 2155304. This source is a calibration target for the XMM-Newton instruments RGS1 and RGS2 (collectively called RGS in the following) and for the Chandra LETG/HRC-S and LETG/ACIS-S combinations (collectively called LETG in the following). Thus, this sight line through the Milky Way is covered by several independent high-resolution X-ray data sets with very high statistical quality. We use these data for studying in detail the Galactic components in this direction via the X-ray absorption lines the Galactic medium imprints on the PKS 2155-304 spectrum. Combining the X-ray and FUV data (see below), we assess the relative importance of collisional ionisation and photoionisation and examine the thermal instability of TTG.

\section{Data}

The detection of most of the Galactic X-ray absorption lines is at the limit of the capabilities of the currently most powerful high-resolution X-ray spectrometers, i.e. XMM-Newton/RGS 
and Chandra/LETG. The exposure time for even the brightest blazars such as PKS 2155-304 should be around a Ms for robust Galactic spectroscopy using absorption lines at the typical equivalent width (EW) level of a few $\mathrm{m} \AA$. Since Ms exposures are observationally too expensive for a single programme, one can only achieve the required sensitivity level by combining a large number of individual observations of the same source. Since PKS 2155-304 is one of the brightest compact X-ray sources with a simple power-law emission spectrum, it has been frequently monitored by XMM-Newton/RGS and Chandra/LETG for calibration purposes. Thus the current PKS 2155-304 observation data base provides an opportunity to advance Galactic $\mathrm{X}$-ray spectroscopy.

The co-addition of a large number of observations, particularly if they span a long period of time, is not trivial. The possible difference in the locations of the bad pixels at different times may create false line-like features if simply co-adding the individual spectra (see Kaastra et al. 2011). This problem could be minimised, in principle, by jointly analysing the individual spectra without co-adding. This approach is problematic as well owing to the complexity of dealing with a large number of involved spectra. Also, the low signal to noise in the individual spectra complicates the estimation of the possible wavelength scale offset. Consequently the modelling of the lines may not be accurate.

\subsection{XMM-Newton/RGS}

In order to overcome these co-addition problems, Kaastra et al. (2015) introduced a new method for combining a large number of RGS observations. These authors used all available data for PKS 2155-304 (see Table A.1) for the purpose of RGS calibration. In the current paper we use this data set. The essential steps of the procedure are as follows:

- The spectra are extracted using the standard SAS tools; we use flux-calibrated spectra.

- Dead channels due to bad pixels were determined for each individual spectrum and their effect was removed before coadding the spectra.

- The time-dependent corrections of the RGS efficiency are implemented by creating an effective area file for each individual spectrum according to its observation date.

- Each spectrum was fitted with a broken power-law model, using a total column density for neutral hydrogen of $N_{\mathrm{H}}=$ $1.24 \times 10^{20} \mathrm{~cm}^{-2}$ (Wakker et al. 2011). To model the effects of dust on the Fe-L and O-K edges we use the "amol" model in SPEX, which uses typical (average) total metal abundances for the amount of $\mathrm{O}, \mathrm{Mg}, \mathrm{Si}$, and $\mathrm{Fe}$ in the gas phase of $0.849,0.187,0.162$, and 0.130 times solar (Pinto et al. 2013; Costantini et al. 2012). We use the properties of $\mathrm{MgSiO}_{3}$ and solid iron to modify the O-K and $\mathrm{Fe}-\mathrm{L}$ absorption edges, since these molecules seem to give the best match to the data (Costantini et al. 2012).

- The final spectrum is constructed by co-adding the weighted residuals at each spectral bin using the individual spectral fits above. Stacking the residuals is equivalent to adding observations, assuming that the continuum modelling is accurate.

The above procedure was carried out separately for the RGS1 and RGS2 data, keeping the 1st and 2nd orders separate. This resulted in four spectra, each with a total exposure time of $\sim 1.2 \mathrm{Ms}$, corrected for the absorption and continuum. The spectra have a constant spectral bin size of $20 \mathrm{m \AA}$, i.e. the energy resolution has been oversampled by a factor of $\sim 3$. Owing to

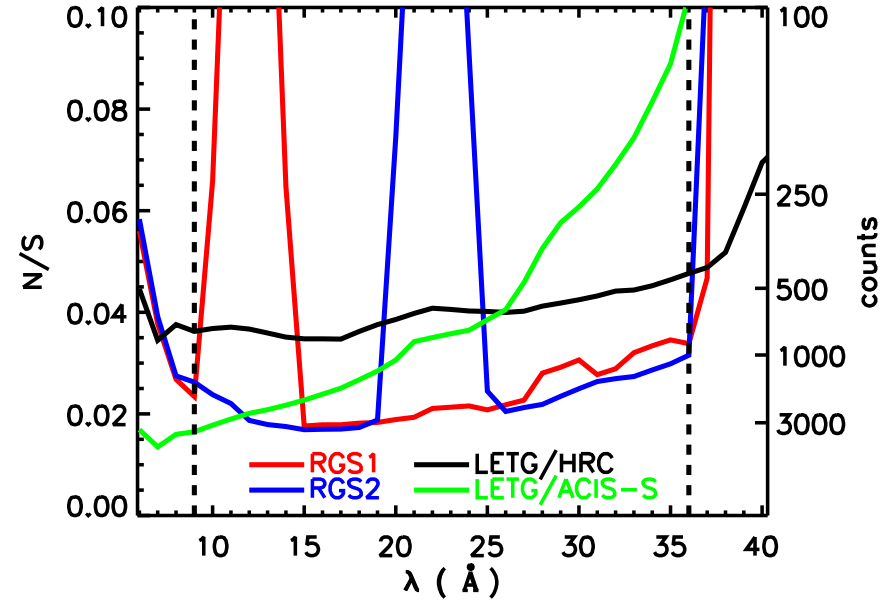

Fig. 1. Ratio of statistical uncertainties to the signal per bin in the coadded PKS 2155-304 observations using RGS1 1st order (red), RGS2 1st order (blue), LETG/HRC-S (black), and LETG/ACIS-S (green). The RGS bin size is $20 \mathrm{~m} \AA$ while LETG bin size is $25 \mathrm{~m} \AA$. The curves have been smoothed strongly for clarity. The dashed lines indicate the waveband selected for the analysis in this paper.

the high exposure time of the spectra, the number of counts in $20 \mathrm{~m} \AA$ channels exceeds a few 1000 and thus the statistical uncertainties are at the $\sim 2-3 \%$ level of the source emission in such bins (see Fig. 1). In the current paper we started from the RGS1 and RGS2 spectra, which were reduced as described above.

Given the large number of counts in our RGS spectral bins (this is also true for LETG; see below) we used $\chi^{2}$ statistics to determine the best-fitting parameters for the baseline absorption line model and the uncertainty in the parameters. The average RGS relative effective area uncertainty from one resolution element to another in our adopted waveband is 2\% (Kaastra 2017) and thus the systematic uncertainties may play a significant role in our analysis. Since the RGS resolution element size is $60 \mathrm{~m} \AA$, a $2 \%$ feature in the continuum corresponds to an equivalent width of $\sim 1 \mathrm{~m} \AA$, which we took as a rough estimate of the calibration uncertainty effect on our EW measurements. We checked the LETG results with a similar level of systematic uncertainty. A more advanced error propagation method is desirable. However, we do not know the probability distributions of the systematic uncertainties in our data. Thus, in the basic analysis we compared results obtained with only statistical uncertainties with those adding the above uncertainties in quadrature to the statistical uncertainties, and we discuss the effects.

We subtracted the background produced by the SAS package. The background flux is $\sim 1 \%$ of the source emission, i.e. at the level of the statistical uncertainties of the total signal. Thus, if the background is uncertain by a given fraction, the background-subtracted signal is uncertain by the same fraction of the statistical uncertainties, i.e. negligible. Thus, we subtracted the standard background (also in the case of LETG) and did not propagate any background-subtraction related uncertainties to our results.

\subsection{Chandra/LETG}

We accumulated all the publicly available Chandra LETG data on PKS 2155-304 (see Table A.2). The total useful exposure times are $\sim 870 \mathrm{ks}$ and $\sim 310 \mathrm{ks}$ for the ACIS-S/LETG and HRCS/LETG combinations. 
Since the data reduction procedure used for our XMMNewton data is not yet applicable to the Chandra data, we reduced the Chandra data with the standard publicly available tools in CIAO 4.7. The data were processed with the standard pipeline (chandra_repro) that generates source spectra, background spectra, and response files, separately for the +1 and -1 order data. We co-added the individual spectra, keeping the ACIS and HRC data separate. In order to reach a sensitivity similar to that of the RGS, we further combined the +1 and -1 order spectra, using the combine_grating_spectra tool. The analysis of Chandra data is therefore performed on two spectra, one containing the LETG/ACIS data and the other containing the LETG/HRC data. We binned the spectra with a constant spectral bin size of $25 \mathrm{m \AA}$, similar to that used for RGS.

\subsection{Sensitivities of different instruments}

When comparing the results obtained with different instruments, we need to consider the variation of their sensitivity and wavelength coverage. The sensitivity of a given combination of a telescope, a spectrometer and a detector to detect absorption lines with a given data set depends primarily on the number of observed counts, i.e. on the product of the effective area, exposure time, and average emission level of PKS 2155-304. All of these factors related to the different data sets used in this work vary and hence the sensitivity varies as well. We estimated the relative sensitivity as a function of wavelength by comparing the ratios of the statistical uncertainties to the signal per adopted bin size (see Fig. 1).

In most of the common wavebands covered with different instruments, the first order data of RGS1 and RGS2 are the most sensitive. In a statistical sense, these data can be used to detect features whose depth exceeds $2-3 \%$ of the continuum level in the 9-36 $\AA$ band we selected for the analysis in this work. The sensitivity of the LETG/HRC-S given purely by the number of counts is smaller than that of the RGS. However, the better energy resolution of the LETG $(F W H M \sim 40 \mathrm{~m} \AA)$ compared to the RGS ( $F W H M \sim 60 \mathrm{~m} \AA)$ improves the relative sensitivity of the LETG/HRC-S towards that of the RGS. The LETG/ACIS-S has a similar sensitivity as RGS at $\lambda=12 \AA$, and it decreases significantly with the wavelength. Since the second order RGS data are of much lower sensitivity and their wavelength coverage is limited, we do not include them in the basic analysis. However, we use the second order data for testing the robustness of our results considering the instrument calibration uncertainties (see Sect. 4.2.1).

\section{Method to find Galactic lines}

In this work we adopted a very conservative approach for the line identification. By using the very high signal-to-noise of our data and the coverage of the important wavelengths by more than one instrument, we attempted to produce a very reliable set of Galactic line measurements in this work.

We tested the presence of the strongest a priori known Galactic absorption lines by examining the wavebands containing the ground-state lines from Verner et al. (1996) and the strongest inner transitions included in the SPEX distribution (Kaastra et al. 1996). We analysed the 9-36 $\AA$ band data of each instrument, i.e. RGS1, RGS2, LETG/HRC-S, and LETG/ACIS-S via the X-ray spectral analysis package SPEX 3.00.00 (Kaastra et al. 1996). However, we did not consider features at the wavelengths of the known bad channels caused by CCD gaps in RGS data or strong instrumental problems at $\lambda \sim 23 \AA$ in both the RGS and LETG (see the XMM-Newton Users Handbook ${ }^{1}$ and the Chandra Proposer's Observatory Guide ${ }^{2}$ ). The only exception is the case for O II, which we treat separately in Sect. 4.3.

We performed the analysis in a narrow band of $\sim 1 \AA$ centred at a given a priori line. We modelled the LETG local blazar emission continuum using a power-law model, allowing both the photon index and the normalisation to be free parameters. In the case of the RGS, the continuum was already modelled and its effect was removed from the data (see Sect. 2.1). Thus, when analysing the lines in the RGS data, we applied a constant model for the continuum, allowing the level to vary from unity to allow for the statistical uncertainties of the continuum.

We fitted the line data with a slab model of SPEX (Kaastra et al. 2002), which calculates the transmission of a thin slab of material whose ion column densities can be varied independently, i.e. the ion ratios are not determined by the ionisation balance. Since we are studying the strongest Galactic lines, with $\tau_{0}$ of order 1, saturation effects are important and we properly take them into account with our procedure; i.e. we do not assume linear growth of $N$ (ion) as a function of EW.

The model produces the Lorentz profile for each transition in the SPEX atomic database for a given ion, including the Auger broadening ${ }^{3}$. Thus, the calculations are also accurate in the case of blended multiplets, such as O IV. The Gaussian component of the Voigt profile is calculated based on the input value of the total velocity dispersion (thermal and non-thermal). Williams et al. (2007) preferred a Doppler parameter of $\sim 50 \mathrm{~km} \mathrm{~s}^{-1}$ when jointly fitting the LETG/ACIS-S and LETG/HRC-S data of the OVII lines in the PKS 2155-304 data. While our data are of higher statistical quality, our attempt to constrain the velocity components did not yield useful results. Thus, when using the Voigt profile of the slab model, we allowed the total velocity dispersion to vary in a range $20-35 \mathrm{~km} \mathrm{~s}^{-1}$, which includes the nonthermal broadening observed in FUV (Wakker et al., in prep.) and thermal broadening of $10 \mathrm{~km} \mathrm{~s}^{-1}$ (the value for oxygen at $\log T(K)=5$ ). If the tested line-like feature was acceptably fitted by a known transition of the expected ion at $z=0$, we upgraded it as a Galactic line candidate.

We then used the best-fit model to obtain the column density of the given ion and the EW of the corresponding line (or blend). If we used a single Gaussian profile for the O I, O IV, O VIII, and C VI multiplets instead, this yielded a significantly lower EW value compared to that obtained with the slab model. This is due to the very high statistical quality of our data rendering the Gaussian approximation of the blended multiplets inaccurate. We obtained the statistical uncertainties of the column densities by $\chi^{2}$ minimisation and consequently the constraints $E W \pm \sigma_{E W \text {,stat }}$ for the equivalent width. We added the approximate calibration uncertainty of $1 \mathrm{~m} \AA$ (see Sect. 2.1) in quadrature to the above uncertainties to obtain the total uncertainties $\sigma_{E W \text {,tot }}$. We used the ratio $E W / \sigma_{E W \text {,tot }}$ as a measure of the detection significance $N_{\sigma}$ (see Table 1 ).

\subsection{Problems and solutions}

The identification of the Galactic lines based on their wavelength is complicated because of the uncertainties in the determination of the wavelength of a given line-like feature in the data. The statistical uncertainties around the peak of the line may cause

\footnotetext{
1 https://heasarc.gsfc.nasa.gov/docs/xmm/uhb/

2 http://cxc.harvard.edu/proposer/POG/html/

3 The damping constant $a$ for the strongest lines (O VII and O VIII Ly $\alpha$ ) is below 0.01 , i.e. the damping-wing effect is negligible.
} 
Table 1. Galactic absorption line measurements.

\begin{tabular}{|c|c|c|c|c|c|c|c|c|c|c|}
\hline \multirow[t]{2}{*}{ Ion } & \multicolumn{2}{|c|}{ Transition } & \multicolumn{2}{|c|}{ RGS1 1st } & \multicolumn{2}{|c|}{ RGS2 1st } & \multicolumn{2}{|c|}{ LETG/HRC-S } & \multicolumn{2}{|c|}{ LETG/ACIS-S } \\
\hline & name & $\lambda_{0}{ }^{a}(\AA)$ & $E W(\mathrm{~m} \AA)^{b}$ & $N_{\sigma}{ }^{c}$ & $E W(\mathrm{~m} \AA)^{b}$ & $N_{\sigma}{ }^{c}$ & $E W(\mathrm{~m} \AA)^{b}$ & $N_{\sigma}{ }^{c}$ & $E W(\mathrm{~m} \AA)^{b}$ & $N_{\sigma}{ }^{c}$ \\
\hline $\mathrm{Ne}$ IX & $1 s-2 p$ & 13.447 & $\mathrm{n} / \mathrm{c}$ & - & $5.1 \pm 1.5$ & 3.4 & $8.0 \pm 2.1$ & 3.9 & $\leq 2.0$ & $\mathrm{n} / \mathrm{d}$ \\
\hline O VII & $1 s-3 b$ & 18.628 & $4.7 \pm 1.5$ & 3.2 & $3.0 \pm 1.5^{d}$ & $2.0^{d}$ & $2.6 \pm 1.9$ & 1.3 & $4.6 \pm 1.5$ & 3.0 \\
\hline O VIII & $1 s-2 p$ & $\begin{array}{l}18.967 \\
18.972\end{array}$ & $9.0 \pm 1.7$ & 5.2 & $5.5 \pm 1.6$ & 5.7 & $7.8 \pm 2.4$ & 3.3 & $5.3 \pm 1.7$ & 3.2 \\
\hline O VII & $1 s-2 p$ & 21.602 & $15.4 \pm 1.5$ & 10.1 & $\mathrm{n} / \mathrm{c}$ & - & $11.3 \pm 2.6$ & 4.3 & $10.0 \pm 1.7$ & 6.0 \\
\hline $\mathrm{Ov}$ & $\begin{array}{l}1 s-2 p \\
1 s-2 p^{2} S\end{array}$ & $\begin{array}{l}22.370 \\
22.571\end{array}$ & $3.0 \pm 1.5$ & 2.0 & $\mathrm{n} / \mathrm{c}$ & - & $3.7 \pm 2.3$ & 1.6 & $\leq 1.1$ & $\mathrm{n} / \mathrm{d}$ \\
\hline OIV & $\begin{array}{l}1 s-2 p^{2} P \\
1 s-2 p^{2} D\end{array}$ & $\begin{array}{l}22.741 \\
22.777\end{array}$ & $7.0 \pm 2.8$ & 2.5 & $\mathrm{n} / \mathrm{c}$ & - & $8.2 \pm 3.1$ & 2.6 & $\leq 4.2$ & $\mathrm{n} / \mathrm{d}$ \\
\hline O I & $1 s-2 p$ & $\begin{array}{l}23.510 \\
23.511\end{array}$ & - & - & - & - & $17.3 \pm 3.3$ & 5.2 & $9.0 \pm 2.0$ & 4.5 \\
\hline N VI & $1 s-2 p$ & 28.788 & $4.5 \pm 2.0^{d}$ & $2.3^{d}$ & $3.1 \pm 1.6$ & 1.9 & $5.0 \pm 2.4$ & 2.1 & $\leq 3.2$ & $\mathrm{n} / \mathrm{d}$ \\
\hline N I & $1 s-2 p$ & 31.286 & - & - & - & - & $10.6 \pm 2.9$ & 3.6 & $\leq 6.1$ & $\mathrm{n} / \mathrm{d}$ \\
\hline C VI & $1 s-2 p$ & $\begin{array}{l}33.734 \\
33.740\end{array}$ & $7.7 \pm 2.6^{d}$ & $2.9^{d}$ & $8.8 \pm 2.0$ & 4.3 & $3.6 \pm 2.6$ & 1.4 & $\leq 6.1$ & $\mathrm{n} / \mathrm{d}$ \\
\hline
\end{tabular}

Notes. ${ }^{(a)}$ A priori values from Verner et al. (1996), Kaastra et al. (1996), and Gu et al. (2005). ${ }^{(b)}$ Equivalent width and total $1 \sigma$ uncertainties (i.e. statistical uncertainties and systematic $1 \mathrm{~mA}$ ) for detected lines. For the non-detections (n/d), the upper $1 \sigma$ limit is given; $\mathrm{n} / \mathrm{c}$ means that the line is not covered by the instrument. ${ }^{(c)}$ Significance of the line detection in terms of $1 \sigma$ uncertainty. ${ }^{(d)}$ The problematic single channel has been omitted when deriving the values.

a random apparent shift of the line centroid. Systematic line shifts may be introduced by the uncertainties due to the limitation of the accuracy of the wavelength scale calibration, i.e. $6 \mathrm{~m} \AA$ for the RGS and $10 \mathrm{~m} \AA$ for the LETG. To evaluate the effect of the apparent centroid shifts, we also fitted the data of our Galactic line candidates using Gaussians with free centroids. A comparison between the fitted and a priori centroid wavelengths of our Galactic line candidates indicated no systematic bias in the wavelength scales. We accepted such lines whose fitted centroid wavelengths were consistent with one of the tested a priori lines, considering the uncertainties of the centroid wavelength at $95 \%$ CL.

In a few cases there were larger shifts in the wavelength, amounting up to $60 \mathrm{~m} \AA$ for O VIII, N VI (RGS1), and O VII Ly $\beta$ (RGS2). This may be related to the observed non-statistical fluctuations in RGS data (Rasmussen et al. 2007). Since these wellknown Galactic lines were securely identified with other instruments, we additionally accepted these into our list of Galactic line candidates. We pay special attention to the effect of these shifts of our reported results below.

In addition to the redshift issue, we have several other problems when identifying and interpreting the line-like features. Since the expected absorbers have line widths that are much smaller than the resolution element, there may be incidences in which the stacking procedure leads to incorrect results. Also, given the high statistical precision of a few per cent of the blazar emission level in our adopted spectral bins with sizes of 20$25 \mathrm{~m} \AA$, we must be very careful not to interpret small residuals due to the possible effective area calibration uncertainties above this level as astrophysical signals. Furthermore, since some of the predicted Galactic lines are relatively weak, we must minimise the possibility of interpreting statistical fluctuations at a few per cent level as celestial. In order to minimise the above effects, we performed screening of the line-like features by applying the following two criteria:

\section{Statistical significance level of $95 \%$.}

We estimated the statistical significance of a given line using the best-fit value and the statistical uncertainties of the equivalent width of the line, keeping the centroid wavelength fixed to an a priori value. We examined a total band width of $\sim 30 \AA$. With a resolution of $\sim 50 \mathrm{~m} \AA$, we have $\sim 600$ resolution elements. Thus, we expect to have $\sim 30$ random events exceeding the $\mathrm{CL}=95 \%$ level $(2 \sigma)$ in the full spectrum. Since we examine features in bands of width $\sim 1 \AA$, the misidentification is possible if one of the $302 \sigma$ fluctuations hits the $1 \AA$ search region. Since our search region is $\sim 3 \%$ of the full band, the expected number of random fluctuations per band is below 1 . Thus, we rejected such lines whose line flux deviates from zero at less than $95 \%$ confidence level, i.e. $2 \sigma$. The only exception is the $1.6 \sigma$ HRC detection of OV, which we additionally accepted (see below).

2. Detection with relevant instruments.

Even if a given Galactic line candidate was detected significantly and consistently with the atomic physics, there is still some probability of a misidentification of a statistical fluctuation or a calibration-related feature as a Galactic line. In order to minimise this probability, we used four independent instruments. If a given astrophysical line is covered with several instruments of comparable sensitivity, it should be detected with all instruments at a comparable confidence level. Given the problems with ACIS detections (see below) we applied this requirement only to RGS and HRC. Given that RGS1 and RGS2 are the most sensitive instruments in most of the studied wavebands, we first required that the given line-like feature in our improved list of Galactic line candidates (see above) must be detected by both RGS1 and RGS2 at $95 \% \mathrm{CL}$, if covered with both. In case the given feature is covered with only one of the RGS units, we conservatively ${ }^{4}$ required that LETG/HRC-S detects the same feature at $95 \% \mathrm{CL}$.

The probability of the null hypothesis, i.e. the no-line assumption about the detection of a feature with two independent instruments, is $(1-0.95)^{2}=0.0025$. Thus, the null hypothesis can be discarded at the $1-0.0025=99.75 \%$ level if

\footnotetext{
4 Since the LETG sensitivity is lower than that of RGS, we may reject
} some of the true Galactic lines with this strict requirement. 


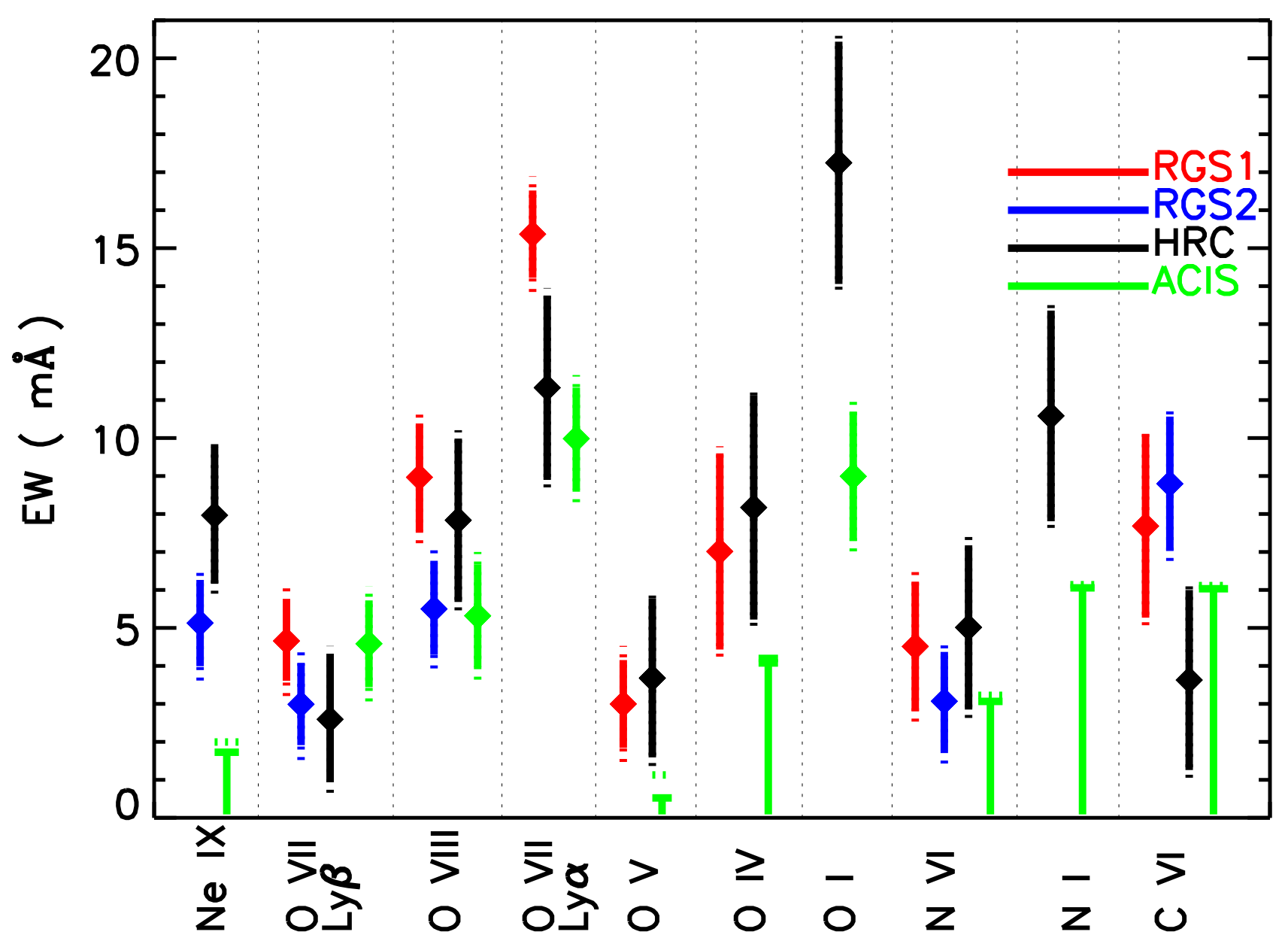

Fig. 2. Equivalent widths and statistical $1 \sigma$ uncertainties of lines (solid lines) detected with RGS1 (red), RGS2 (blue), LETG/HRC-S (black), and LETG/ACIS (green). For the non-detections, we report the $1 \sigma$ upper limits. The dotted lines indicate the effect of adding the $2 \%$ systematic uncertainties.

two independent lines are detected at the $2 \sigma$ CL. If the feature also passed this test, we upgraded it as a true Galactic line.

\section{Detections and identifications}

The above procedure resulted in detections of 10 Galactic absorption lines identified with their ionic species and transitions (see Table 1 and Figs. 2-12). As expected by the sensitivity calculations (see Fig. 1), the RGS and HRC detected lines with equivalent width exceeding $\approx 2 \mathrm{~m} \AA$. Considering all the instruments, there were 25 independent significant detections. The RGS1 and RGS2 $1 \sigma$ EW constraints for all four lines in common agreed. In most cases the RGS and HRC measurements yielded EW values consistent for a given line within the uncertainties. The only exception is N VI, whose measurements by RGS2 and HRC differ marginally. The systematic uncertainty level of $1 \mathrm{~m} \AA$ causes a very small effect on the uncertainties (see Fig. 2). In summary, the possible systematic uncertainties of the effective area calibration of RGS and HRC do not yield significant biases for the EW measurement.

However, the ACIS seems to differ systematically from the other instruments. We discuss this issue next, together with other sources of systematic uncertainties.

\subsection{Instrumental issues}

\subsubsection{Centroid shifts}

When using the best-fit centroid wavelengths to identify the Galactic lines (Sect. 3), we noted significant shifts for the centroids of the O VIII and N VI (RGS1) and O VII Ly $\beta$ (RGS2) lines from the a priori values. It is not clear whether the true EW is recovered better by using the apparent or the a priori wavelength for the line centroid. Thus, we examined the EW values obtained by keeping the centroid free or fixing to a priori value. The change was not significant, implying that whatever is causing the shift of these lines does not have significant effect on our EW measurements.

\subsubsection{Non-statistical fluctuations}

In case of RGS1 lines N VI and C VI and RGS2 O VII Ly $\beta$ line, the best-fit model yielded significant residuals (see Figs. 10, 12 and 4). In very deep exposures such as those in this work, occasionally non-statistical fluctuations become visible (see Rasmussen et al. 2007). Usually, these are confined to excursions in a single narrow wavelength bin (significantly narrower than the spectrometer resolution). A single such excursion seems to be visible in the data of all the above lines between wavelengths 28.782-28.795, 33.721-33.74, and 18.660-18.680 А. 


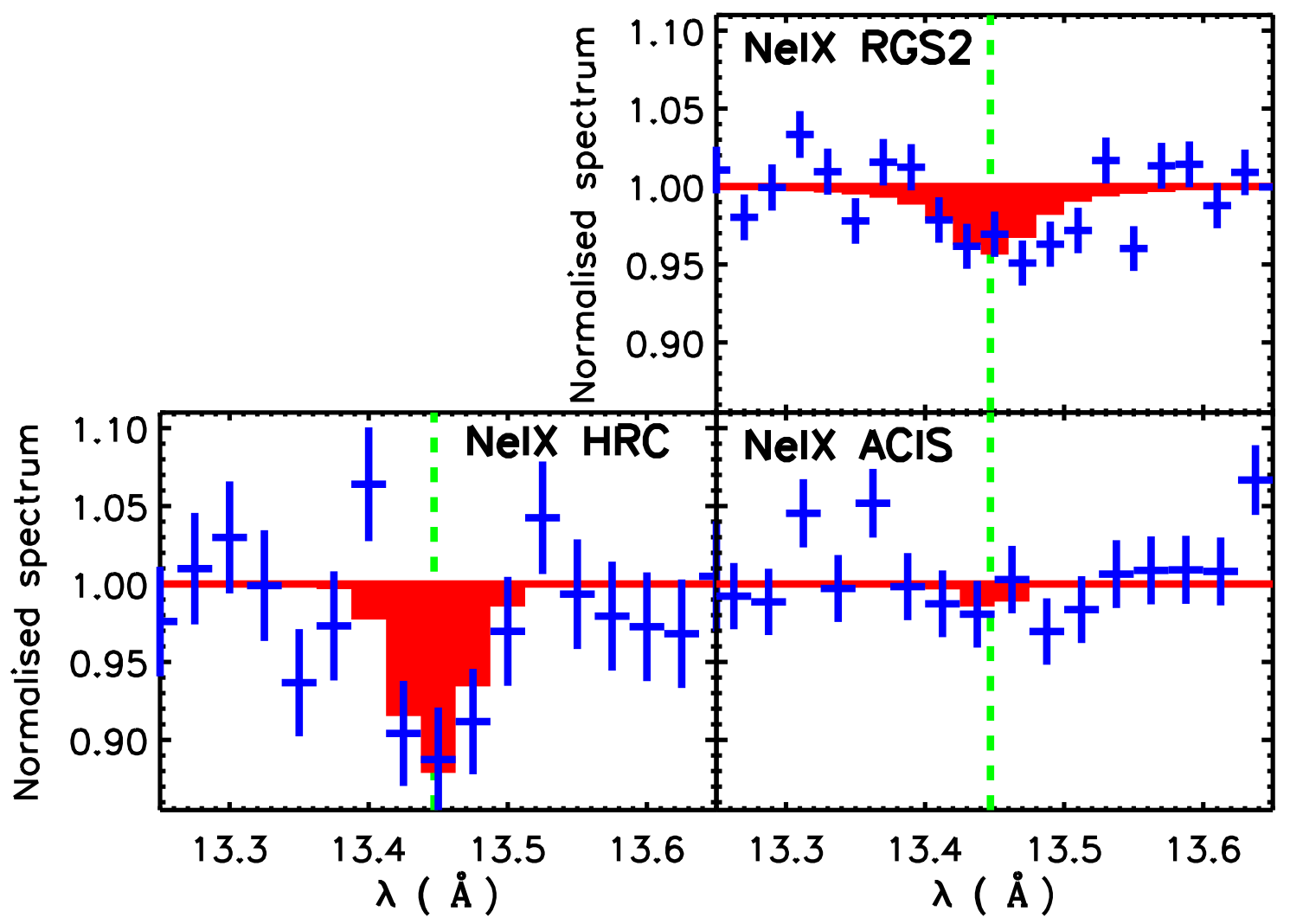

Fig. 3. Normalised data (blue crosses) and best-fit models (solid red lines) of the Ne IX line for RGS2 (upper right), LETG/HRC-S (lower left), and LETG/ACIS-S (lower right). The RGS1 does not cover these wavelengths. The normalisation is carried out by dividing the spectra by the power-law component. The error bars reflect only the statistical uncertainties.

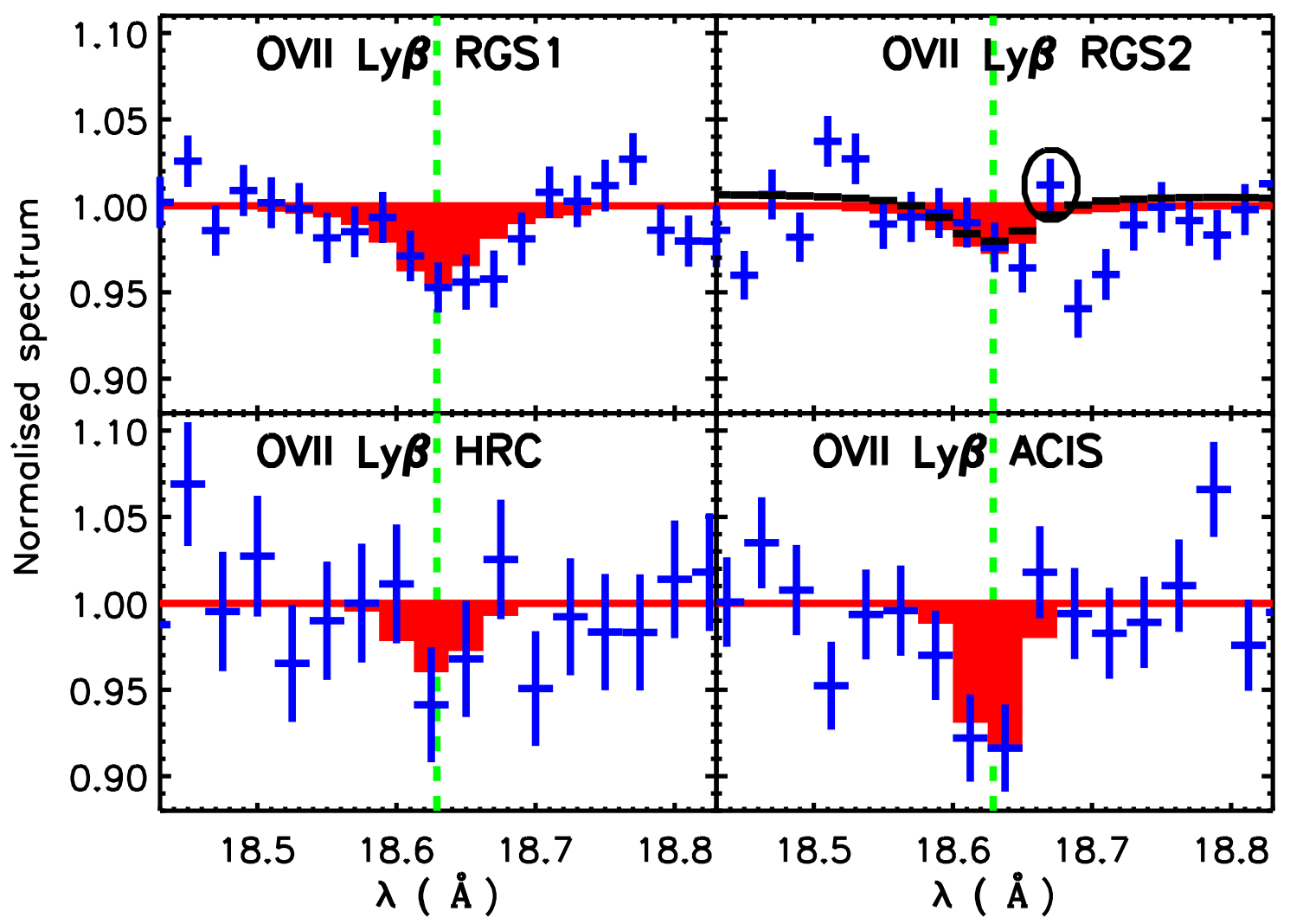

Fig. 4. As in Fig. 3, but for O VII Ly $\beta$ transition line. The black line in the upper right panel indicates the best-fit line profile when excluding the strongly deviant channel (surrounded by a black ellipse). 


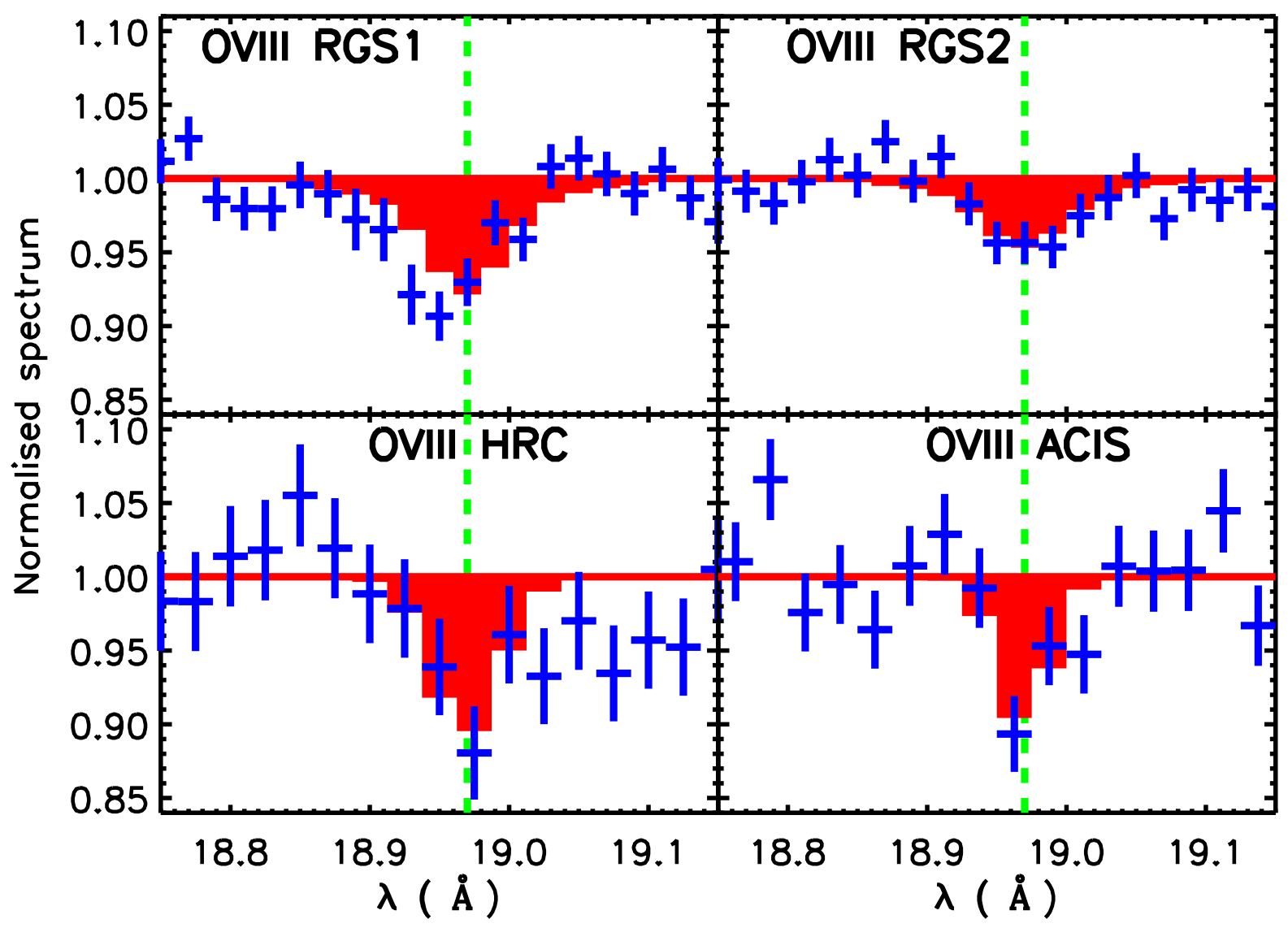

Fig. 5. As in Fig. 3, but for the O VIII Ly $\alpha$ transition line.

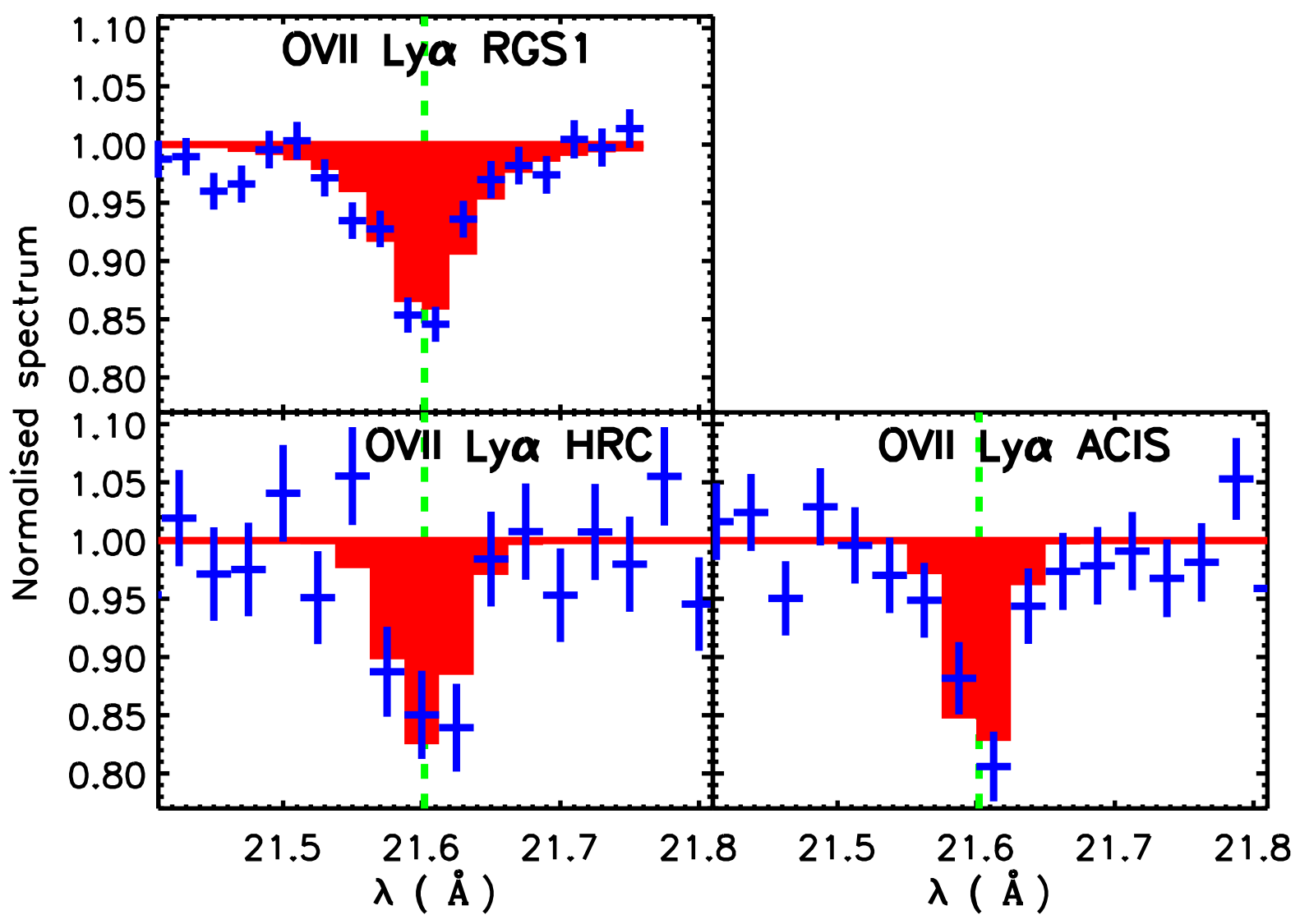

Fig. 6. As in Fig. 3, but for the O VII Ly $\alpha$ line. The RGS2 does not cover these wavelengths. 


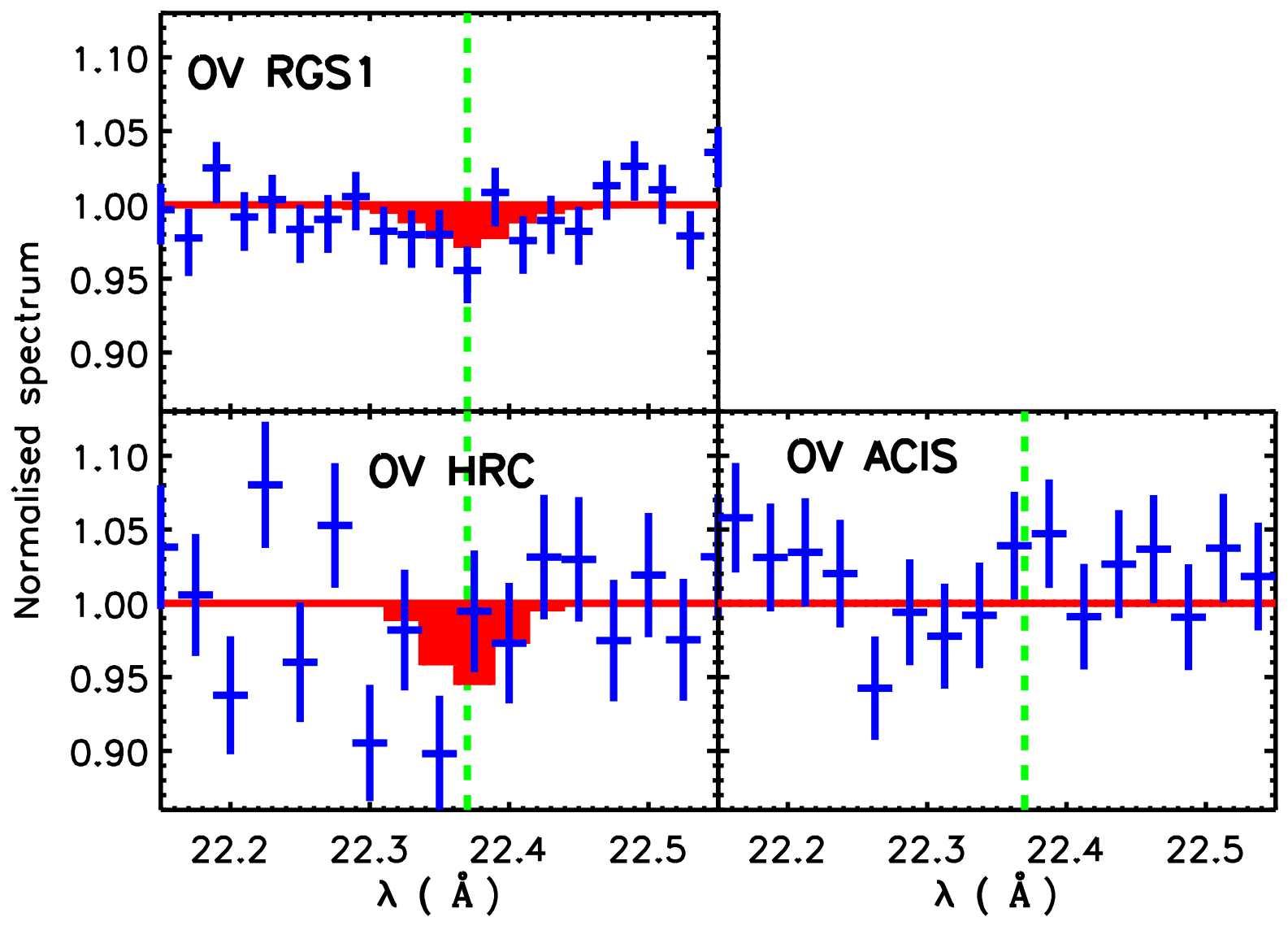

Fig. 7. As in Fig. 3, but for the O v line. RGS2 does not cover these wavelengths.

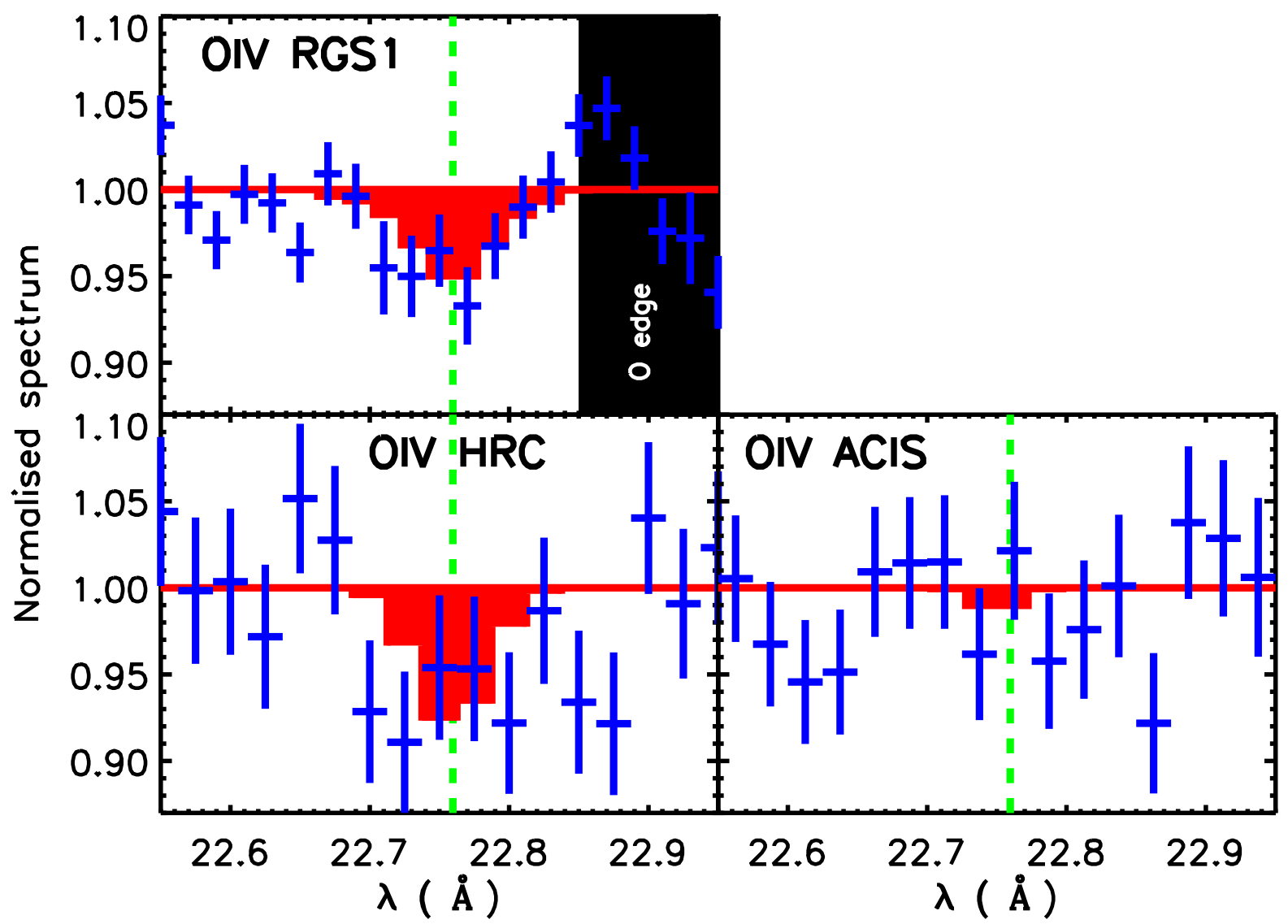

Fig. 8. As in Fig. 3, but for the O IV line. RGS2 does not cover these wavelengths. The RGS1 oxygen edge is indicated with the black box. 


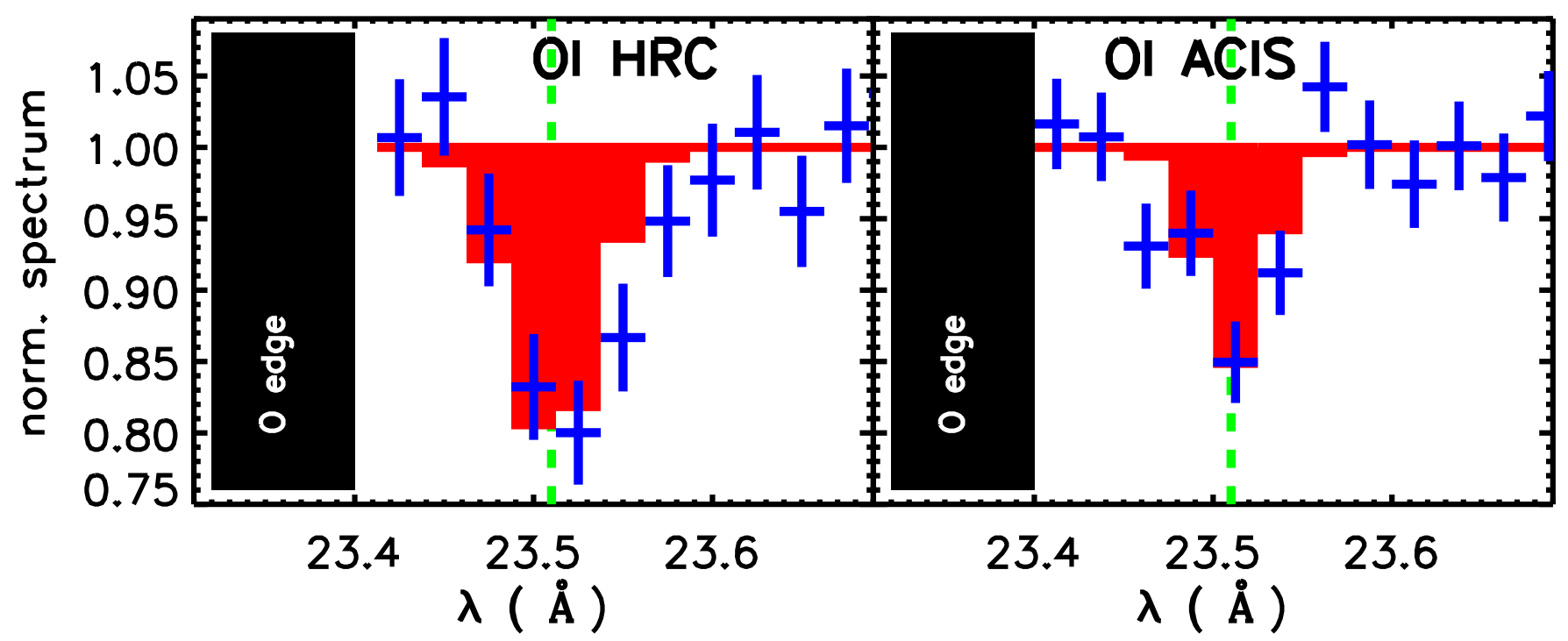

Fig. 9. As in Fig. 3, but for the O I line. The black boxes indicate the omitted wavelengths due to the instrumental oxygen edge.

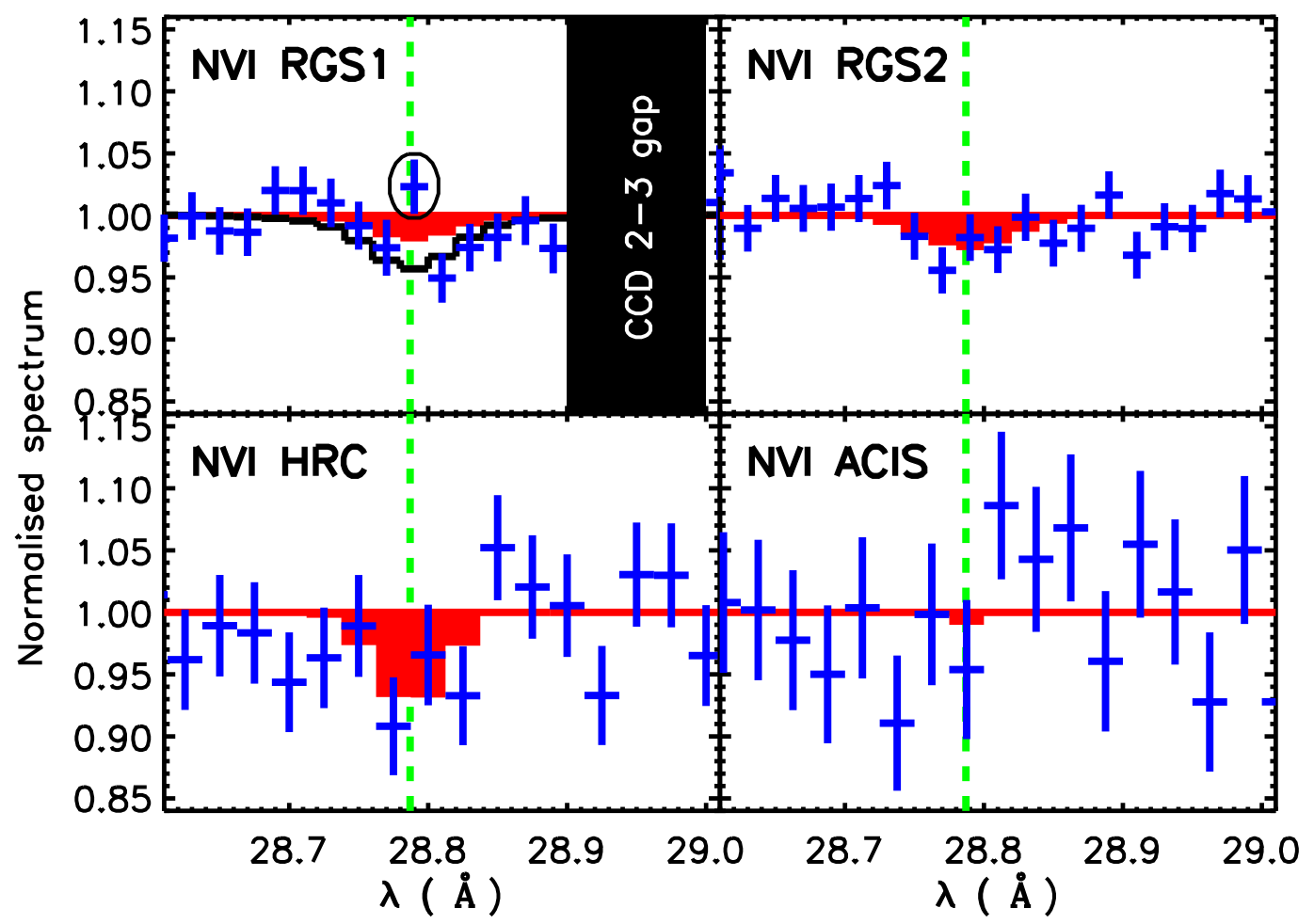

Fig. 10. As in Fig. 3, but for the N vI line. The gap between the RGS1 CCD 2 and CCD 3 gaps is denoted with the black box. The black line in the upper left panel indicates the best-fit line model when excluding the strongly deviant channel (surrounded by a black ellipse).

When ignoring these, the EW of RGS1 NVI and C VI lines increased significantly into better agreement with RGS2. The RGS2 OVII Ly $\beta$ was not affected. In the following, we exclude these problematic channels.

\subsubsection{LETG/ACIS-S negative bias}

At $\lambda \leq 25 \AA$ the LETG/ACIS-S should be more sensitive than LETG/HRC-S and comparable to RGS (see Fig. 1). In fact, the detection confidences of the O VII and O VIII $1 \mathrm{~s}-2 \mathrm{p}$ lines for ACIS are greater than for HRC (see Table 1).

However, ACIS did not detect the Ne IX, Ov, OIV, N VI, $\mathrm{N}$ I, and C VI lines, which were detected with other instruments covering these wavelengths. Also, the ACIS detection of O I yielded a value of $\sim 8 \mathrm{~m} \AA$ for $\mathrm{EW}(\mathrm{OI})$, which is lower by a factor of 1.9 than that of the HRC (see Fig. 9 and Table 1). These measurements point to a negative bias of several $\mathrm{m} \AA$ in LETG/ACIS-S EW measurements. This effect may be related to the pixelation of the ACIS CCDs, which slightly undersamples the Chandra PSF. While the native spectral bins used in the standard data processing are smaller than the energy resolution, the final effective resolution may be worse than that of HRC, washing out the weakest features.

On the other hand, the LETG/ACIS did detect the O VII Ly $\alpha$, $\mathrm{O}$ VII Ly $\beta$, and O VIII Ly $\alpha$ lines and yielded EW consistent with the RGS2 and HRC for these lines. Thus, the systematic effect is 


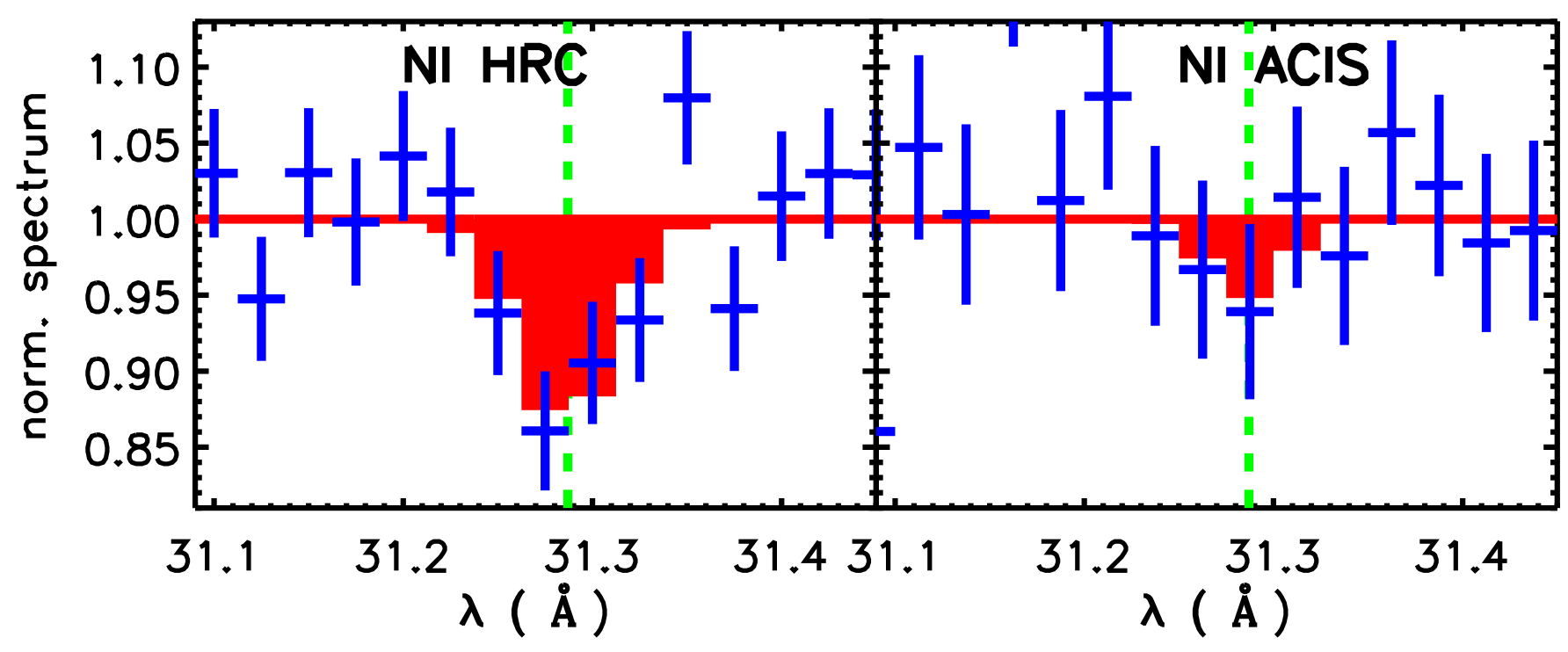

Fig. 11. As in Fig. 3, but for N I line.

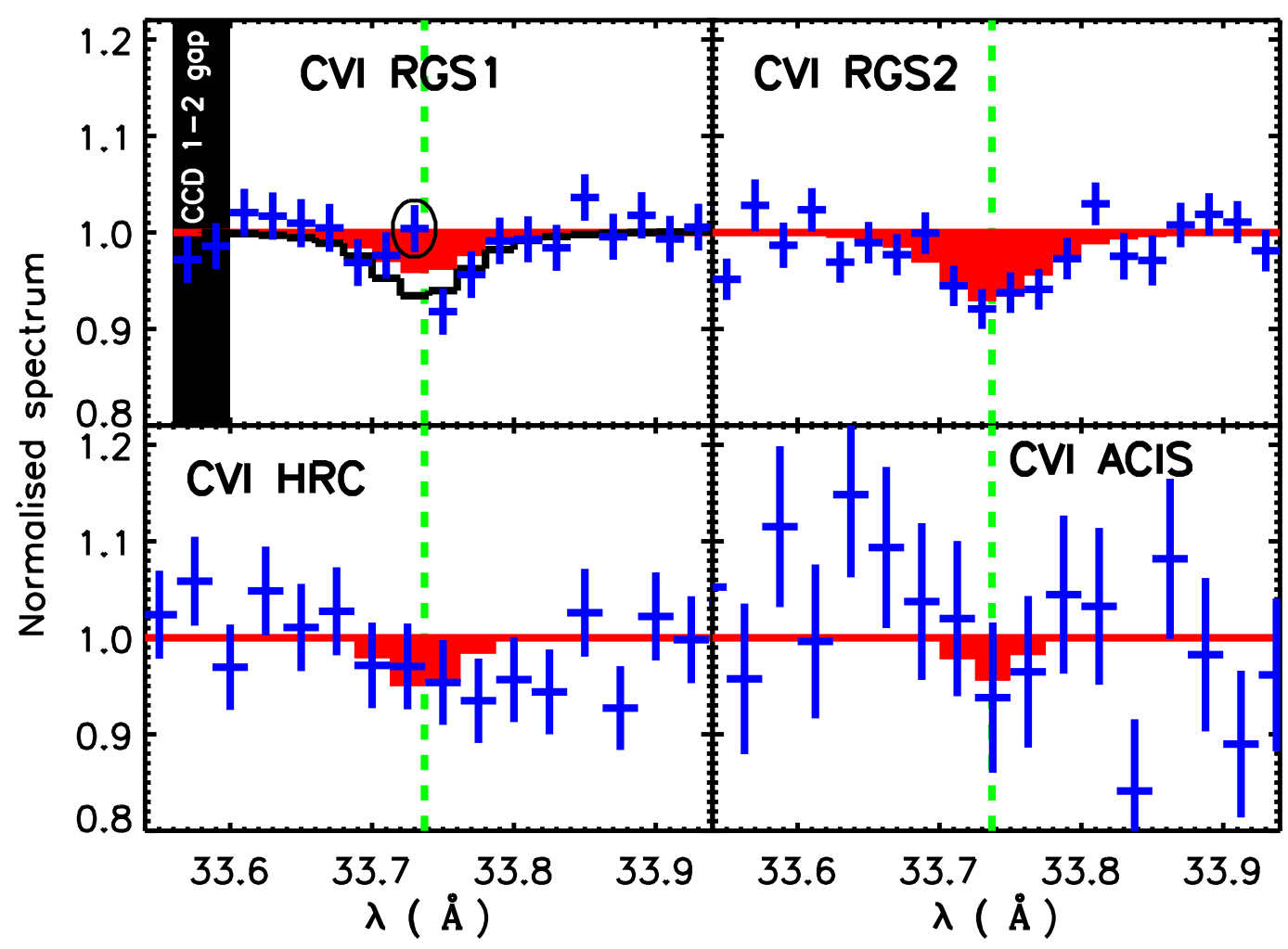

Fig. 12. As Fig. 3, but for the C VI line. The gap between the RGS1 CCD 1 and CCD 2 is denoted with a black box. The black line in the upper left panel indicates the best-fit line model when excluding the strongly deviant channel (surrounded by a black ellipse).

hard to understand and take properly into account when measuring absorption lines with LETG/ACIS-S.

\subsection{Results}

Our very strict criteria for the identification of the Galactic lines were needed to minimise the possibility of misidentification of noise as Galactic lines. The downside is that we may omit some interesting weaker lines due to the Milky Way (e.g. Nicastro et al. 2016) or the intervening extragalactic warm hot integalactic medium (e.g. Fang et al. 2007; Williams et al. 2007). We will address these in a future paper.

Considering the temperatures at which the absorption lines from different ions reach their maximal equivalent width (e.g. Kaastra et al. 2008), our detections indicated three distinct absorbing components: neutral disk (ND), hot halo (HH), and transition temperature gas (TTG).

- ND: the OI and $\mathrm{NI}$ ions (detected with the LETG) are obviously associated with the neutral $k T \sim 10^{-3} \mathrm{keV}$ $(\log T(\mathrm{~K}) \sim 4)$ Galactic disk (ND) absorber. 

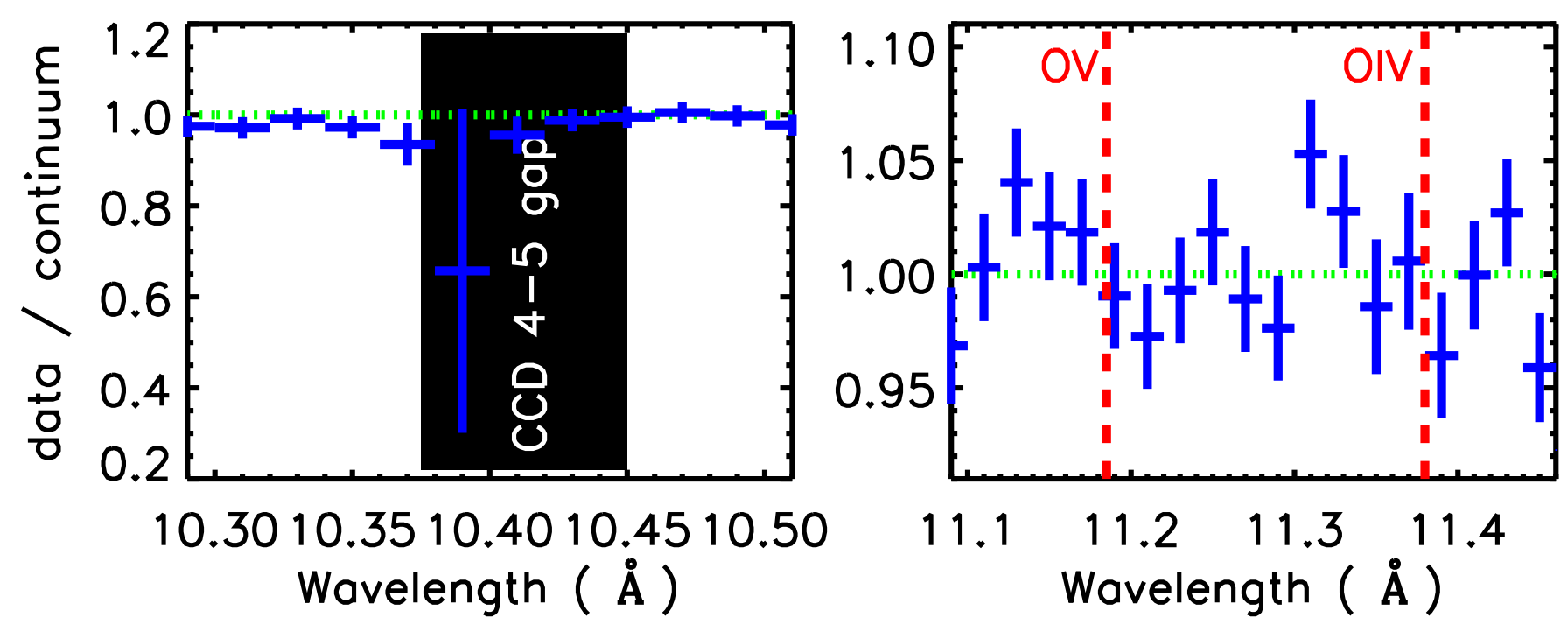

Fig. 13. RGS1 second order data of PKS 2155-304, divided by the continuum, are shown with blue crosses in different wavebands (different panels). The black box is placed at 0.5 times the wavelengths of the CCD gaps in the RGS1 first order (left panel). The vertical lines are placed at 0.5 times the wavelengths of O IV and O V (right panel).

- $H H:$ an absorber with $k T \sim 0.1 \mathrm{keV}(\log T(\mathrm{~K}) \sim 6)$ is indicated by the lines from Ne IX, O VII, O VIII, N VI, and C VI. We attribute this to the hot part of the Galactic halo $(\mathrm{HH})$.

- TTG: the detection of the lines due to the inner shell $1 \mathrm{~s}-2 \mathrm{p}$ transitions of $\mathrm{OV}$ and O IV with RGS1 and LETG/HRC-S (see Figs. 7 and 8) revealed the existence of an absorber with $k T \sim 0.01 \mathrm{keV}(\log T(\mathrm{~K}) \sim 5)$, i.e. TTG. This is the first time that this component has been seen in X-ray absorption and the first time that O IV and OV have been definitely detected in the Milky Way.

A study of the RGS effective area calibration around the oxygen edge (de Vries et al. 2003) reported an absorption feature at $22.77 \AA$ in the RGS1 spectra of PKS 2155-304 and Mrk 421. Since the same feature was indicated in the Chandra LETG data of the same sources, and it was absent in the RGS data of the Galactic sources Sco-X1 and 4U 0614+091, de Vries et al. concluded that the feature is a true Galactic signal in the directions towards PKS 2155-304 and Mrk421. They suggested that this feature is due to absorption by O IV. Our analysis confirms this suggestion, and we also detect $\mathrm{O} v$ from the same absorber.

\subsubsection{Second order test for the TTG lines}

Given that our Galactic O V and O IV X-ray absorption detections are the first secure detections in the literature (except for the suggestion in de Vries et al. (2003)), and that for the first time we use these lines to detect the Galactic transition temperature gas in X-rays, we used the second order RGS1 data for additional testing and confirmation. We used the fact that a detector position of a given wavelength of the first order corresponds exactly to half of that wavelength in the second order. Thus, a given instrumental artifact at a detector, which gives a feature at the first order wavelength $\lambda_{1}$, should yield a feature in the second order spectrum at wavelength $\lambda_{2}=0.5 \times \lambda_{1}$. In fact, the dead areas between the CCD gaps in the 1st order RGS1 spectra imprint a sudden drop of counts in narrow bands in the second order data at half the wavelengths of the first order CCD gaps (see Fig. 13).

We found that at the wavelengths 0.5 times of those of $\mathrm{O} I \mathrm{~V}$ and $\mathrm{OV}$, the second order data are consistent with a flat continuum, i.e. strong detector artifacts erroneously causing the O IV or O v detections are ruled out (see Fig. 13).

\subsubsection{O IV and the oxygen edge}

As stated before, we have not considered features at wavebands significantly affected by known instrumental effects (CCD gaps and $\mathrm{O}$ edge). Given that the wavelength of the OIV line $(\lambda \approx$ $22.76 \AA$ ) is close to the band affected by the RGS1 instrumental oxygen edge, we made an additional check. The instrumental efficiency of RGS1 varies rapidly by $\sim 20 \%$ in narrow wavebands at $\lambda=22.8-23.3 \AA$ (see Fig. 14). In this band the residuals indicate calibration-related features at the $5 \%$ level of the continuum. The second order data also indicate features at these wavelengths, demonstrating that uncertainties of the efficiency calibation around the oxygen edge may amount to deviations at the level of $5 \%$ of the continuum. At the wavelengths of the O IV line (22.7-22.8 $\AA$ ), the RGS1 efficiency is flat and thus likely more accurately calibrated. In fact, the data are well fitted with the power-law + narrow Gaussian model with no significant scatter (see Figs. 8 and 14). Thus, the calibration uncertainties due to the oxygen edge around the O IV line are likely smaller than $5 \%$, i.e. they cannot produce an artificial line-like feature similar to the feature we associate with O IV.

\section{3. $0 \| 1 s-2 p$}

Inspired by the recent report on the detection of Galactic O II 1s2 p line (Nicastro et al. 2016) with LETG in the PKS 2155-304 sight line we checked this line in our spectra. We had excluded these wavelengths $(\lambda \sim 23.35 \AA)$ from our previous analysis due to the coincidence of the wavelengths of O II $1 \mathrm{~s}-2 \mathrm{p}$ with an instrumental feature in RGS1 and LETG. de Vries et al. (2003) argued that in both instruments there is O I $1 \mathrm{~s}-2 \mathrm{p}(\lambda=23.5 \AA)$ absorption, shifted to $23.35 \AA$ due to O I in the solid compounds of the instruments (metal oxide or water ice). This feature is included in the effective area calculations of both RGS and LETG.

We fitted a narrow Gaussian $\left(F W H M=10 \mathrm{~km} \mathrm{~s}^{-1}\right)$, plus a local power law in the $\sim 1 \AA$ band centred at $\lambda=23.35 \AA$, 


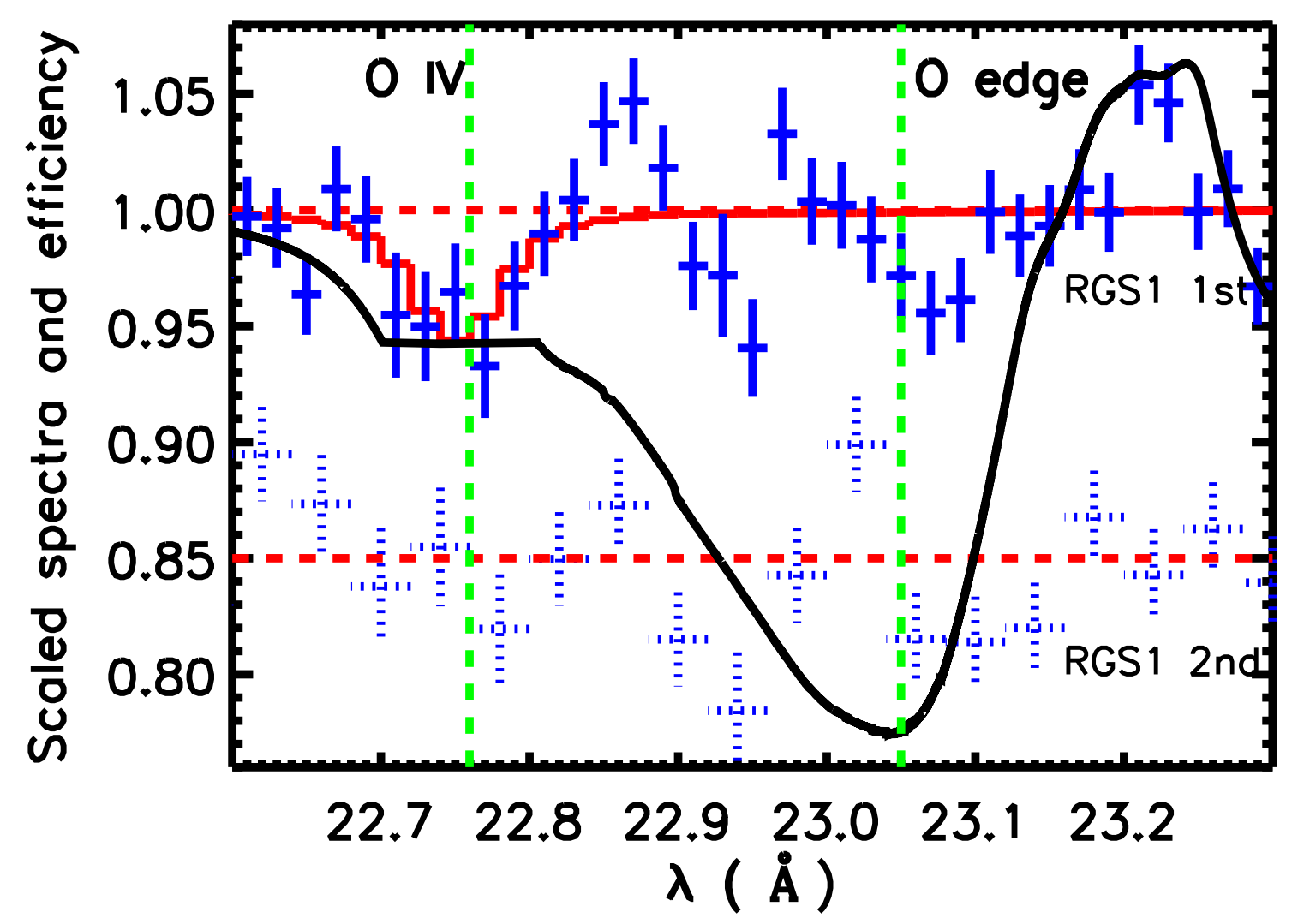

Fig. 14. RGS1 first order data (blue solid crosses) and best-fit power law + narrow Gaussian fit (solid red line), divided by the power-law component in the waveband containing the O IV line and the oxygen edge (green dashed lines) The RGS1 second order data (blue dotted crosses) are shown at wavelengths 2 times the original wavelengths, scaled arbitrarily for display purposes. The black curve indicates the RGS1 instrumental efficiency from de Vries et al. (2003), scaled to unity at $\lambda=22.6 \AA$.

independently to RGS1, HRC and ACIS data. We ignored the waveband 23.45-23.60 $\AA$ in order not to bias the continuum due to the OI line. In the case of the RGS1 and HRC, the data indicated significant excess absorption on top of the instrumental feature (see Fig. 15). The Gaussian modelling yielded inconsistent values $E W(\mathrm{RGS} 1)=3.2 \pm 1.3 \mathrm{m \AA}$ and $E W(\mathrm{HRC})=11.6 \pm$ $2.5 \mathrm{~m} \AA$ for the excess. The ACIS yielded only an upper limit $E W($ ACIS) $\leq 0.5 \mathrm{~m} \AA$ (Table 2), which is inconsistent with the other instruments. This is different from Nicastro et al. (2016) who reported that HRC and ACIS yielded consistent EW for O II. The average value of EW(O II $1 \mathrm{~s}-2 \mathrm{p})$ measured with HRC and ACIS in Nicastro et al. (2016) is $8.6 \pm 1.1 \mathrm{m \AA}$, which is inconsistent with our RGS1 and ACIS values. The origin of the discrepancy between our measurements and those of Nicastro et al. (2016) is unclear.

A very generous maximum EW for O II at $\lambda=23.7 \AA$ can be estimated by assuming that all oxygen for the amount of $N(\mathrm{HI})$ in the PKS 2155-304 sight line $\left(\sim 10^{20} \mathrm{~cm}^{-2}\right)$ is in the form of O II. Assuming additionally a turbulent broadening of $60 \mathrm{~km} \mathrm{~s}^{-1}$ yields $E W(\mathrm{O}$ II $) \leq 9 \mathrm{~m} \AA$. However, in reality the radiation field conditions in the disk and inner halo indicate that the $\mathrm{O}$ II to O I column density ratio is much below 1 . Thus, the expected EW for O II is below $1 \mathrm{~mA}$, which is much smaller than that derived with RGS1 and HRC.

Our low ACIS value compared to that of RGS1 could be understood by the underperformance of ACIS we found above. However, the significantly high HRC EW measurement compared to RGS1 is inconsistent with our work. Namely, six of
Table 2. O II measurements.

\begin{tabular}{lc}
\hline \hline & $\begin{array}{c}E W(\mathrm{O} \text { II } 1 \mathrm{~s}-2 \mathrm{p}) \\
\mathrm{m} \AA\end{array}$ \\
\hline RGS1 & $3.2 \pm 1.3$ \\
$\mathrm{HRC}$ & $11.6 \pm 2.5$ \\
$\mathrm{ACIS}-\mathrm{S}$ & $\leq 0.5$ \\
$\mathrm{~N}^{a}{ }^{a}$ & $8.6 \pm 1.1$ \\
\hline
\end{tabular}

Notes. ${ }^{(a)}$ N16: average of HRC and ACIS-S as reported by Nicastro et al. (2016).

the secure Galactic lines are detected with both RGS1 and HRC, within statistical precision of $2 \mathrm{~m} \AA$, and there is no instance in which HRC would yield significantly higher EW than RGS1.

Thus, assuming that the calibration of the instrumental feature at $\lambda=23.35 \AA$ is accurate, our measurements indicate that the reported OII 1s-2p signal (Nicastro et al. 2016) is not due to a constant astrophysical source, as it should yield a constant EW for the same source as measured with different instruments. Also our HRC and RGS1 measurements of the EW are much bigger than allowed by the above H I consideration. Thus, it is likely that the signal is at least partly due to inaccuracies of the calibration of the instrumental feature at $23.35 \AA$. The simultaneous calibration of the instrumental feature and the measurement of the possible astrophysical O II signal requires more work. 


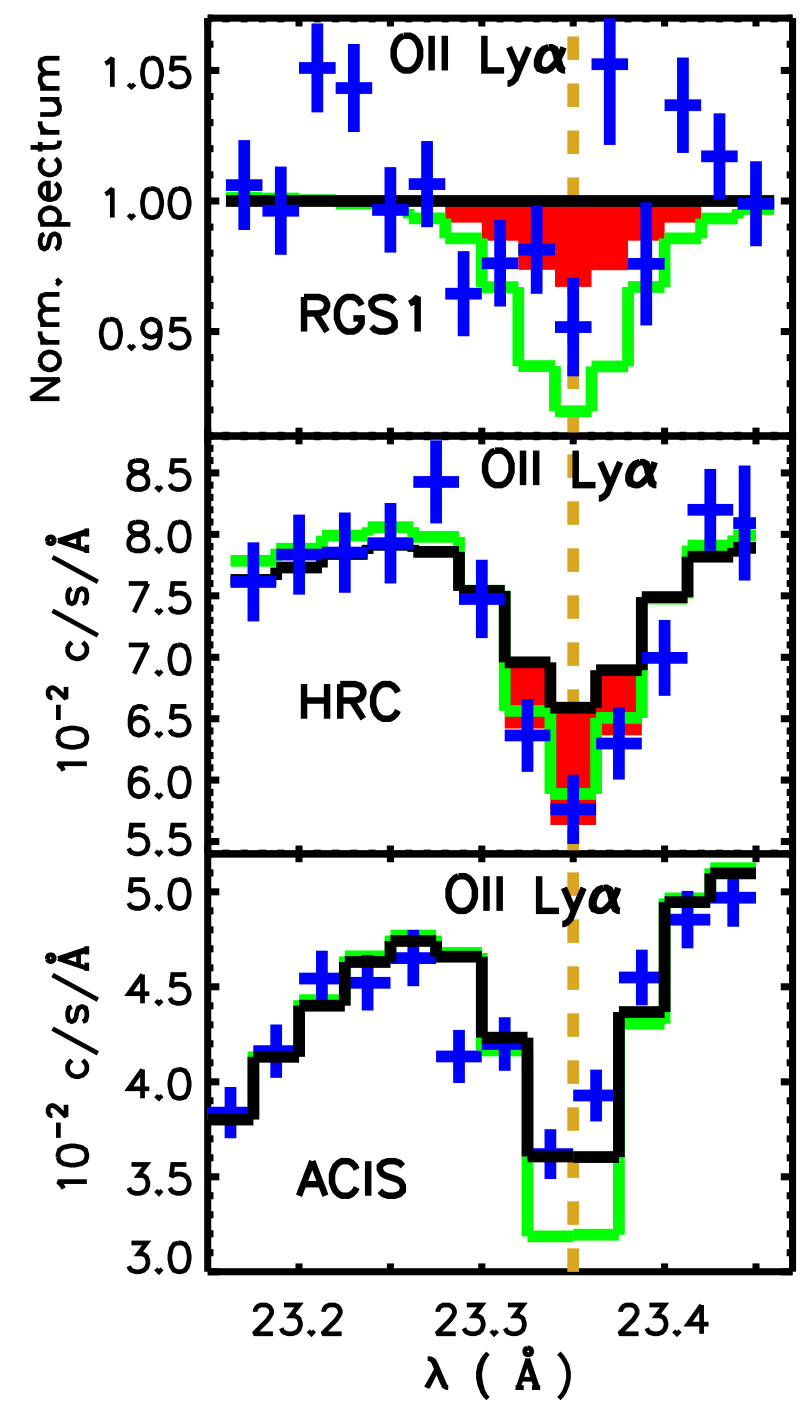

Fig. 15. Blue crosses show the data around the O II 1s-2p wavelengths for RGS1 (upper panel), HRC (middle panel), and ACIS (lower panel). In case of HRC and ACIS we show the data without dividing with the continuum while this is not possible with RGS data owing to the special data processing. The black lines indicate the continuum, which in case of HRC and ACIS is convolved with the responses. The red areas (for RGS1 and HRC) indicate the Gaussian model contribution above the continuum. In the case of ACIS it is negligible. The green curves indicate the model from Nicastro et al. (2016).

\section{Column densities without thermal equilibrium assumptions}

The line fitting procedure described above also yielded the column densities of the ions producing the lines (see Sect. 3). As indicated by the consistency of EW measurements above, for each ion, RGS1, RGS2, and HRC yielded consistent column densities (see Table 3 and Fig. 16).

In the case of the TTG, the RGS1 constraints at the $1 \sigma$ (statistical and systematic) uncertainty level $\left(E W_{\text {OIV }}=7.0 \pm 2.8 \mathrm{~m} \AA\right.$ and $\left.E W_{\mathrm{OV}}=3.0 \pm 1.5 \mathrm{m \AA}\right)$ correspond to $\log N\left(\mathrm{OIV}\left(\mathrm{cm}^{-2}\right)\right)=$ $15.61_{-0.21}^{+0.15}$ and $\log N\left(\mathrm{OV}\left(\mathrm{cm}^{-2}\right)\right)=15.17_{-0.45}^{+0.26}$.

The upper limits of the column densities derived using the ACIS data are inconsistent with the constraints derived with other instruments (see Table 3 and Fig. 16) for several ions.
Table 3. Ion column densities.

\begin{tabular}{lcccc}
\hline \hline \multirow{2}{*}{ Ion } & \multicolumn{4}{c}{$\log N\left(\mathrm{~cm}^{-2}\right)^{a}$} \\
& RGS1 & RGS2 & HRC & ACIS \\
\hline Ne IX & - & $15.96_{-0.35}^{+0.40}$ & $16.59_{-0.46}^{+0.48}$ & $\leq 15.37$ \\
O VII $^{b}$ & $16.24_{-0.33}^{+0.18}$ & $15.93_{-0.45}^{+0.28}$ & $15.86_{-0.80}^{+0.27}$ & $16.07_{-0.18}^{+0.13}$ \\
O VIII & $16.03_{-0.17}^{+0.16}$ & $15.71_{-0.23}^{+0.18}$ & $15.92_{-0.19}^{+0.18}$ & $15.70_{-0.17}^{+0.14}$ \\
O V & $15.17_{-0.45}^{+0.26}$ & - & $15.28_{-0.45}^{+0.28}$ & $\leq 14.40$ \\
O IV & $15.61_{-0.15}^{+0.15}$ & - & $15.68_{-0.22}^{+0.16}$ & $\leq 15.38$ \\
O IV (CIE) & $15.56_{-0.21}^{+0.21}$ & - & $15.51_{-0.35}^{+0.23}$ & - \\
O I & - & - & $16.54_{-0.35}^{+0.11}$ & $16.19_{-0.11}^{+0.10}$ \\
N VI & $15.05_{-0.38}^{+0.25}$ & $14.84_{-0.46}^{+0.25}$ & $15.23_{-0.41}^{+0.33}$ & $\leq 14.87$ \\
N I & - & - & $15.81_{-0.12}^{+0.15}$ & $\leq 15.56$ \\
C VI & $15.34_{-0.24}^{+0.19}$ & $15.41_{-0.16}^{+0.13}$ & $14.97_{-0.51}^{+0.25}$ & $\leq 15.22$ \\
\hline
\end{tabular}

Notes. ${ }^{(a)}$ The values are obtained by fitting the data with the slab model, i.e. without an assumption about the ionisation balance, except for O IV, for which we also show the value obtained under CIE assumption. The uncertainties include the systematic uncertainty of $1 \mathrm{~m} \AA$ and the allowance of $v_{\text {tot }}=20-35 \mathrm{~km} \mathrm{~s}^{-1}$. ${ }^{(b)}$ The value for RGS1, RGS2, and $\mathrm{HRC}$ is the weighted mean of the $\operatorname{Ly} \alpha$ and $\operatorname{Ly} \beta$ measurements. RGS2 only covers the $\operatorname{Ly} \beta$ line.

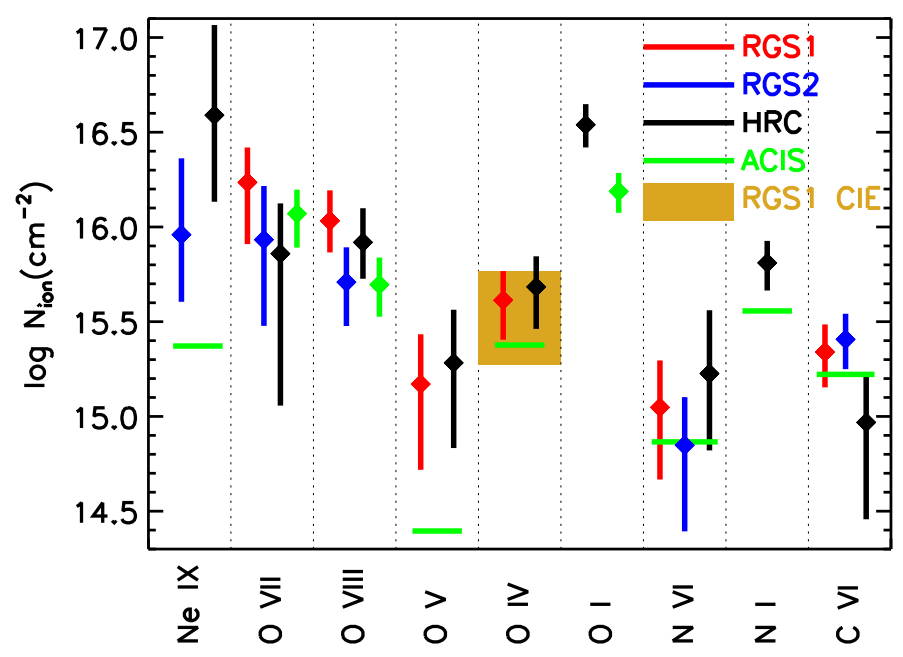

Fig. 16. Best-fit values and confidence intervals including statistical and systematic uncertainties at $1 \sigma$ level of the column densities of the ions producing the reliable detected Galactic lines (diamonds and vertical bars). The upper limits obtained with ACIS are indicated with green horizontal bars. Values are derived without an assumption about the ionisation balance (solid lines) except for O IV, for which we additionally show the RGS1 values assuming CIE (filled rectangle).

Owing to several non-detections of the lines, we did not use the ACIS data in the spectral analysis (Sect. 9.1).

\section{Collisional ionisation equilibrium versus photoionisation}

\subsection{Method}

The two competing ionisation processes in the case of TTG are photoionisation and collisional ionisation. It is likely that collisional ionisation equilibrium (CIE) holds for the $\mathrm{HH}$ due to its relatively high temperature. The column densities of ions occurring in TTG may be affected by photoionisation if the ionisation parameter was sufficiently high. Before using the ion column 
Table 4. COS column densities and velocities for the absorbing components in the PKS 2155-304 sight line (Wakker et al. 2012, for high ions), Wakker et al., in prep. for low ions, and H I column density from FUSE.

\begin{tabular}{ccccccccc}
\hline \hline$v\left(\mathrm{~km} \mathrm{~s}^{-1}\right)$ & & & \multicolumn{2}{c}{$\log N\left(\mathrm{~cm}^{-2}\right)$} & & Si IV & Al II \\
\hline-270 & H I & C II & C IV & O VI & Si II & Si III & Si \\
-232 & $14.0 \pm 0.3$ & $<12.55$ & $13.36 \pm 0.04$ & $13.22 \pm 0.08$ & $<11.96$ & $12.15 \pm 0.10$ & $12.34 \pm 0.22$ & $<12.00$ \\
-134 & $16.25 \pm 0.25$ & $13.72 \pm 0.03$ & $13.26 \pm 0.05$ & $13.26 \pm 0.08$ & $<11.96$ & $11.93 \pm 0.13$ & $12.37 \pm 0.22$ & $<11.98$ \\
MWY $^{a}$ & 20.11 & $>14.94$ & $13.70 \pm 0.04$ & $14.04 \pm 0.04$ & $14.15 \pm 0.02$ & $>13.69$ & $13.07 \pm 0.02$ & $>13.33$ \\
\hline
\end{tabular}

Notes. ${ }^{(a)}$ The low-velocity component.

densities for estimating the temperature of TTG we made an attempt to determine the importance of photoionisation.

For this, we used the CLOUDY (Ferland et al. 1998) code to assess the circumstances under which photoionisation might be important for the creation of O IV and O V. The CLOUDY code assumes pure photoionisation and predicts the ionic column densities as a function of distance, metallicity and the ionisation parameter $U=n(\gamma) / n(\mathrm{H})$, i.e. the ratio of number densities of the ionising photons to hydrogen. Assuming the values for the distance and metallicity, matching the FUV-measured ion column densities (see below) with the CLOUDY model yields the estimate for $U$. An additional estimate of $n(\gamma)$ (see below) then yields the estimate for the hydrogen number density $n(\mathrm{H})$, and consequently for the number densities of metals. The measurement of hydrogen column density $N(\mathrm{H} \mathrm{I})$ (see below) then yields the path length $L=N(\mathrm{H}) / n(\mathrm{H})$ of the absorber and thus calculates the predictions for the column densities of the elements not used above. In practice, we use the above procedure to compute the predictions for the column densities of O IV and OV.

We then compare the predictions with our X-ray measurements of O IV and O V column densities as derived from RGS1 data without an assumption about the ionisation equilibrium (see Table 3). The predictions vary depending on the assumed distance $D$ and thus we test different scenarios for the TTG origin: Galactic halo/disk $(D=1 \mathrm{kpc})$, vicinity of the Galactic halo $(D=$ $20 \mathrm{kpc}$ ) and Local Group environment ( $D=200 \mathrm{kpc})$.

To characterise the ionising radiation field in these different scenarios (i.e. the $n(\gamma)$ value at the different distances), we followed the method of Fox et al. (2005). They used the Haardt \& Madau (2001) model of the intensity and spectrum of the extragalactic ionising radiation, combined with a model of the Galactic contribution, with the latter varying as a function of the location of the cloud in the Galactic halo.

\subsection{Velocity structure}

A problem in the assessment of the role of the photoionisation is that in the direction of PKS 2155-304, FUSE and STIS spectra show a complicated kinematic structure (Sembach et al. 1999; Wakker et al. 2003, 2012). Sembach et al. (1999) reported the Si II and C IV and a non-detection for C II in the PKS 2155-304 sight line. They concluded that the clouds have $\log U \sim-3.0$, density $\log n\left(\mathrm{~cm}^{-3}\right) \sim-4$ and a size of several kpc. They interpreted these components as analogous to those seen in damped Ly-alpha absorbers that originate in the diffuse outer halos of galaxies. However, they did not include the Galactic contribution to the ionising radiation field. If they had included this contribution, it would imply $\log n \sim-2$ and a cloud size of a few $100 \mathrm{pc}$.

Sembach et al. (1999) also derived a metallicity of $\log Z=-0.5$ for these clouds, but this is rather uncertain.
To make a prediction for ionic column densities we do our calculations assuming possible metallicities $\log Z=0,-0.5$ and -1.0 .

We improve significantly upon the previous results by using a more sensitive (as yet unpublished) COS spectrum, obtained in 2012, which reveals even more absorption components than were analysed in the earlier papers. A full analysis of the COS spectra that shows these absorption lines lies outside the scope of this paper. However, we present the column densities measured for the different ions in Table 4, since they are relevant for understanding the $\mathrm{O} I \mathrm{~V}$ and $\mathrm{O}$ v column densities.

In the PKS 2155-304 sight line, there are two components at velocities $<75 \mathrm{~km} \mathrm{~s}^{-1}$ and three at high velocity (i.e. HVCs and high-velocity clouds). The low-velocity components are seen in low, intermediate, and high ions. The low ions are likely associated with the nearby neutral disk and lower halo, which has a scale height of $3 \mathrm{kpc}$ (Savage \& Wakker 2009; Wakker et al. 2012). The high-ionisation lines (C IV, O VI) most likely have a mixed origin, where O VI probably originates in interfaces between hot and cool gas in the lower and upper galactic halo. Some of the C IV is also produced in these regions, but another (unknown) fraction is due to photoionisation. For the modelling discussed below we assume the extreme situation in which all $\mathrm{C}$ IV is due to photoionisation. A smaller fraction would make the conclusions we derive stronger.

The two high-velocity components centred at $v=-270$ and $-232 \mathrm{~km} \mathrm{~s}^{-1}$ are only detected in lines of highly ionised atoms (O VI, C IV, Si III, and Si IV). The component at $-134 \mathrm{~km} \mathrm{~s}^{-1}$ shows these ions and the low-ionisation lines of O I, C II, Si II, and AlII. The FUSE spectrum (Wakker et al., in prep.) also shows Lyman series absorption for these clouds, which allows the derivation of the H I column densities shown in Table 4.

Since the O IV and O V X-ray absorption is not kinematically resolved, we cannot determine which fraction of the absorption is associated with the low-velocity or high-velocity gas. We consider various possibilities below.

\subsection{High-velocity cloud origin}

The location of the high-velocity clouds in the direction towards PKS 2155-304 is uncertain. Sembach et al. (1999) suggested they might be rather distant (a few $100 \mathrm{kpc}$ ) clouds in the Local Group environment. Alternatively, they might be in the vicinity of the Galactic halo at a distance of tens of kpc. Considering their velocity and likely density it seems unlikely that they are in the lower halo.

We first examined the lower distance scenario in which the $-134 \mathrm{~km} \mathrm{~s}^{-1}$ cloud is near the Milky Way, at a distance of $20 \mathrm{kpc}$ with C II, C IV, Si II, Si III, and Si IV column densities measured for this component (see Table 4). We adopted $\log \mathrm{H} I=16.25$ from the FUSE spectrum (Wakker et al., in prep.) and assumed 


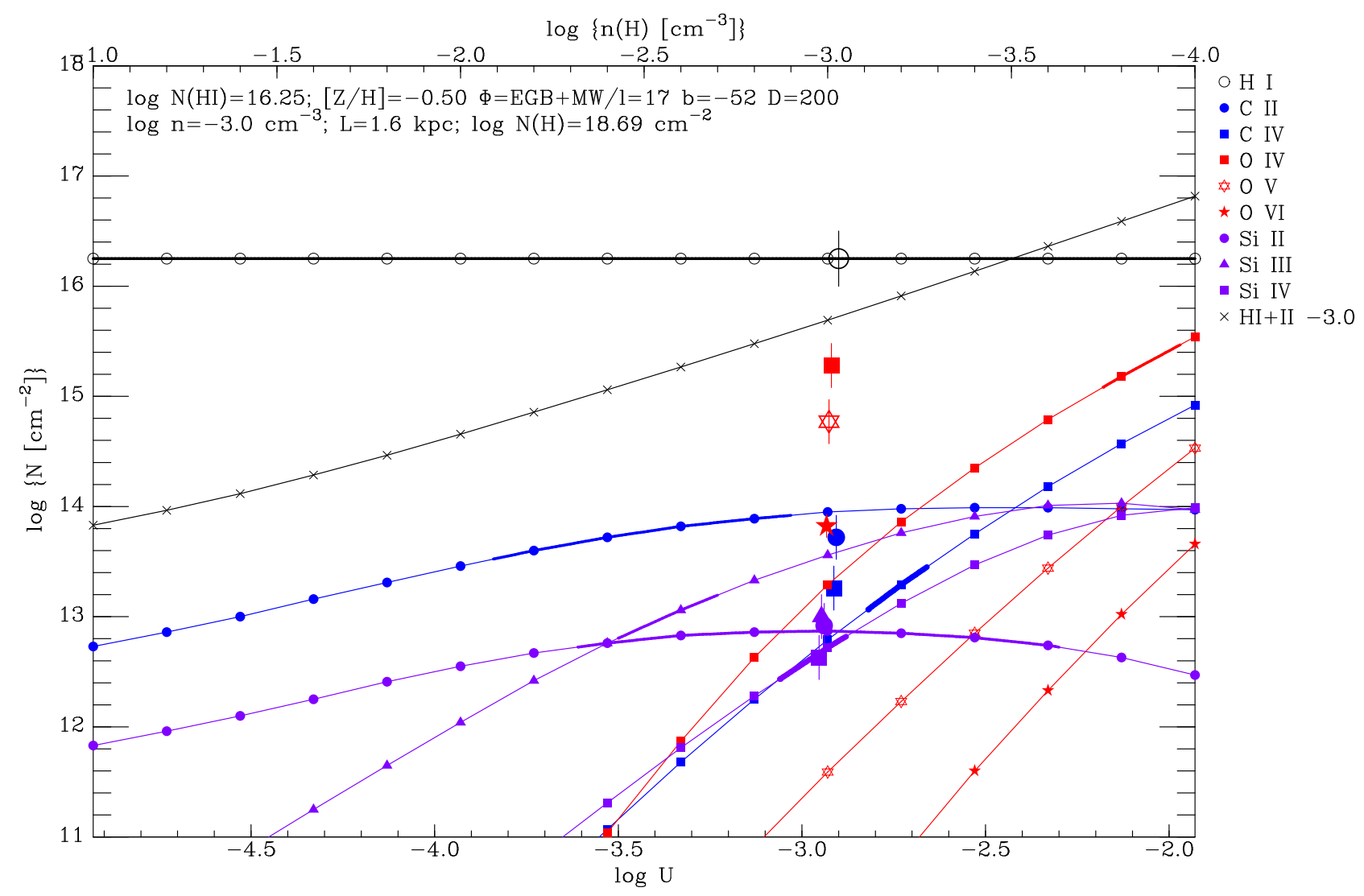

Fig. 17. Predicted ionic column densities as a function of ionisation parameter U,calculated for a location $200 \mathrm{kpc}$ from the Sun in the direction of PKS2155-304. Black lines and points indicate hydrogen; blue lines and points indicate carbon, red indicate oxygen, and purple indicate silicon. Circles indicate C II and Si II, triangles for Si III, squares indicate C IV and O IV, and stars indicate O V. The observed values are indicated by the larger symbols near $\log U=-3.0$, at which value both C IV and Si IV match the observations. The thick parts of the lines show where the prediction matches the range of observed values.

a metallicity $\log Z=-0.5$. In this case the CLOUDY modelling yields an ionisation parameter $\log U \sim-2.7$ and implies a total hydrogen column $\log N(\mathrm{H})\left(\mathrm{cm}^{-2}\right)=18.94$. This can be turned into the hydrogen number density and path length using the intensity of the ionisation radiation field. In our model, which is identical to that of Fox et al. (2004), this yields $\log n(\mathrm{H})\left(\mathrm{cm}^{-3}\right) \sim$ -2.0 and a path length of $280 \mathrm{pc}$ at this location $(20 \mathrm{kpc}$ from the Sun in the direction $l, b=17,-52$ ).

Assuming instead that the absorber is at the distance of $200 \mathrm{kpc}$, i.e. at the Local Group environment, we obtained $\log U \sim-3.0, \log N(\mathrm{H})\left(\mathrm{cm}^{-2}\right)=18.69, \log n(\mathrm{H})\left(\mathrm{cm}^{-3}\right) \sim-3.0$, and a path length $\sim 1.6 \mathrm{kpc}$ (see Fig. 17).

In both cases $N(\mathrm{O} \mathrm{IV})$ and $N(\mathrm{OV})$ are underpredicted by a factor of 100-1000 (see Fig. 17). Below the He II limit (54.4 eV), the Galactic contribution to the intensity of the ionising radiation is about a factor 10 larger than the extragalactic contribution, while between 54 and $\sim 100 \mathrm{eV}$ it is a factor 2 larger. Thus, at larger distances the radiation field is harder and the relative amount of $\mathrm{O}$ IV and $\mathrm{O}$ V (ionisation potentials 54.9 and $77.4 \mathrm{eV}$ ) is larger. However, even including the strong X-ray intensity present in some models of the EGB (see e.g. Shull et al. 2014 for a comparison) is insufficient to produce the amount of observed O IV, O v, and O VI through photoionisation.

We then experimented by varying the metallicity between $\log Z=0$ and -1 and replacing the column densities with those measured for the -270 and $-232 \mathrm{~km} \mathrm{~s}^{-1}$ clouds. Consequently, the cloud densities varied in the range $\log n\left(\mathrm{~cm}^{-3}\right)=-3.2$ to -1.8 and the cloud sizes ranged from 0.1 to $5.1 \mathrm{kpc}$. Yet, the O IV and $\mathrm{OV}$ underprediction remained at the same level as above.

Alternately, explaining $N(\mathrm{O}$ IV $)$ and $N(\mathrm{OV})$ as due to pure photoionisation would require a much higher ionisation parameter ( $\log U>-2.0$; implying a cloud size of $30 \mathrm{kpc}$ or larger). However, given the observed value of $N(\mathrm{HI})$, in this case the predicted values of $N(\mathrm{CIV})$ and $N(\mathrm{Si} I \mathrm{~V})$ are more than two orders of magnitude larger than the observed values.

In summary, it seems likely that the observed C II, C IV, Si II, Si III, and Si IV originate in a sub-kpc size photoionised condensation in the Galactic vicinity or Local Group environment, but it is not possible that the X-ray-observed O IV and O V column densities can be produced in such a cloud.

\subsection{Galactic disk or halo origin}

Trying to assess whether photoionisation could be responsible for producing $\mathrm{O}$ IV and $\mathrm{OV}$ in the Galactic disk or halo is more difficult because thecorresponding column densities of kinematically resolved ions are undetermined. Most of the H I emission detected at $21 \mathrm{~cm}$ originates within a few $100 \mathrm{pc}$ from the disk, where the volume density is so high that $\mathrm{O} I$ is the dominant oxygen ion while $\mathrm{O} I V$ and $\mathrm{OV}$ is mostly absent. It is also not possible to determine the fraction of the column density any other ion that comes from the lower halo rather than the disk.

Our approach is to assume that O IV and O v originate in the same volume and see whether the observed column densities can be consistent with some value of $\mathrm{N}(\mathrm{H})$ and other ionic column 


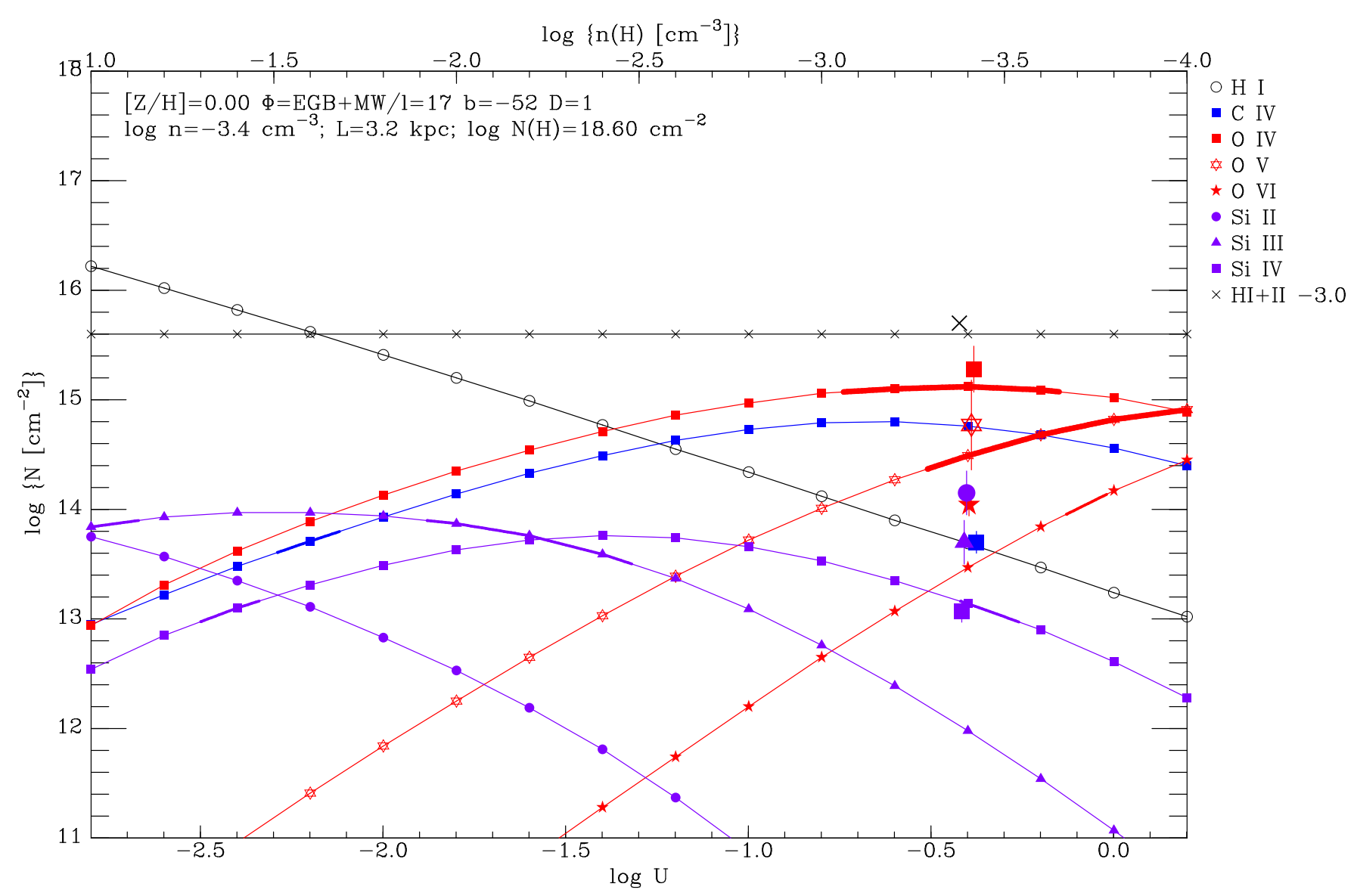

Fig. 18. As in Fig. 17, but for the Galactic halo or disk case.

densities for the ionising radiation field near the disk. Since there are two components with similar column densities in ions such as C IV and O VI (Wakker et al. 2012), we use half the observed value for the modelling.

As shown in Fig.18, matching the observed O IV/O V column densities and their ratio then is possible with an ionisation parameter $\log U \sim-0.4$, a total hydrogen density $\log n\left(\mathrm{H}\left(\mathrm{cm}^{-3}\right)\right) \sim$ -3.6 , and a column density $\log N\left(\mathrm{H}\left(\mathrm{cm}^{-2}\right)\right) \sim 18.6$. This implies a path length of $3.2 \mathrm{kpc}$, which would imply that the Galactic halo is filled with O IV/O V containing gas without much density fluctuations. Such a situation can also fit the Si IV and O VI column densities, but it underpredicts $N$ (Si III) by two orders of magnitude, which could come from a different phase.

However, this situation also predicts that $\log N(\mathrm{C}$ IV $)=14.7$, a factor 10 larger than is observed. Therefore, assuming that $\mathrm{O}$ IV, O V originate in photoionised gas in the Galactic disk/halo environment is inconsistent with the data.

Thus, we rule out a significant contribution by photoionisation to the O IV and O V column densities measured in our X-ray spectra.

\section{Thermal instability}

Before reporting the CIE modelling of the TTG in detail, we study here the effects of the possible deviation of TTG from the ionisation equilibrium. The CIE modelling indicated a temperature $\log T(\mathrm{~K}) \sim 5$ for TTG (see Sect. 9), at which temperature the gas is in a thermally unstable phase, i.e. the heating and cooling timescales of TTG are shorter than the ionisation or recombination time scales (e.g. Gnat \& Sternberg 2007). Consequently, the TTG ions are associated with flows of heating or cooling material, as assumed in the Galactic Fountain models (see Sect. 9). Thus, the CIE-derived temperature may be biased, given the suitable conditions.

Our X-ray measurements of O IV and OV column densities, derived from EW measurements without assumptions about the equilibrium, cannot alone constrain the non-CIE models. Thus, we combined them with the FUV measurement of $\log N(\mathrm{O}$ VI $)=14.31 \pm 0.10$ in the PKS 2155-304 sight line (Wakker et al. 2012). In the case of O VI about half the column density comes from the gas near $v \sim 0 \mathrm{~km} \mathrm{~s}^{-1}$, while the other half is associated with high-velocity clouds in the line of sight. Although the O IV and O V X-ray absorption lines are unresolved, we assume a similar combination of low-velocity and high-velocity cooling parcels of transition temperature gas as in FUV.

We used the non-equilibrium cooling models of Gnat \& Sternberg (2007) to calculate the column density ratios of OIV, OV, and OVI as a parcel of gas cools down from $T=10^{6}$ to $10^{4.4} \mathrm{~K}$ (see Fig. 19). Between $\log T(\mathrm{~K})=6$ and 5.3 the cooling is slow and the ionisation equilibrium is maintained. At $\log T(\mathrm{~K}) \sim 5.3$ the cooling turns isobaric, i.e. the density of a TTG cloud increases as the temperature decreases. Consequently, the O IV/O VI column density ratio falls below the CIE prediction for a given $\mathrm{O} I V / \mathrm{O} V$ column density ratio. This reflects the "frozen-in" O VI that cannot recombine fast enough as the temperature of the gas drops quickly. When the total column density $N(\mathrm{H})$ exceeds

$N(\mathrm{H})=1.2 \times 10^{19} \mathrm{~cm}^{-2} \times\left[\frac{T}{10^{5} \mathrm{~K}}\right]^{2.0}$ 


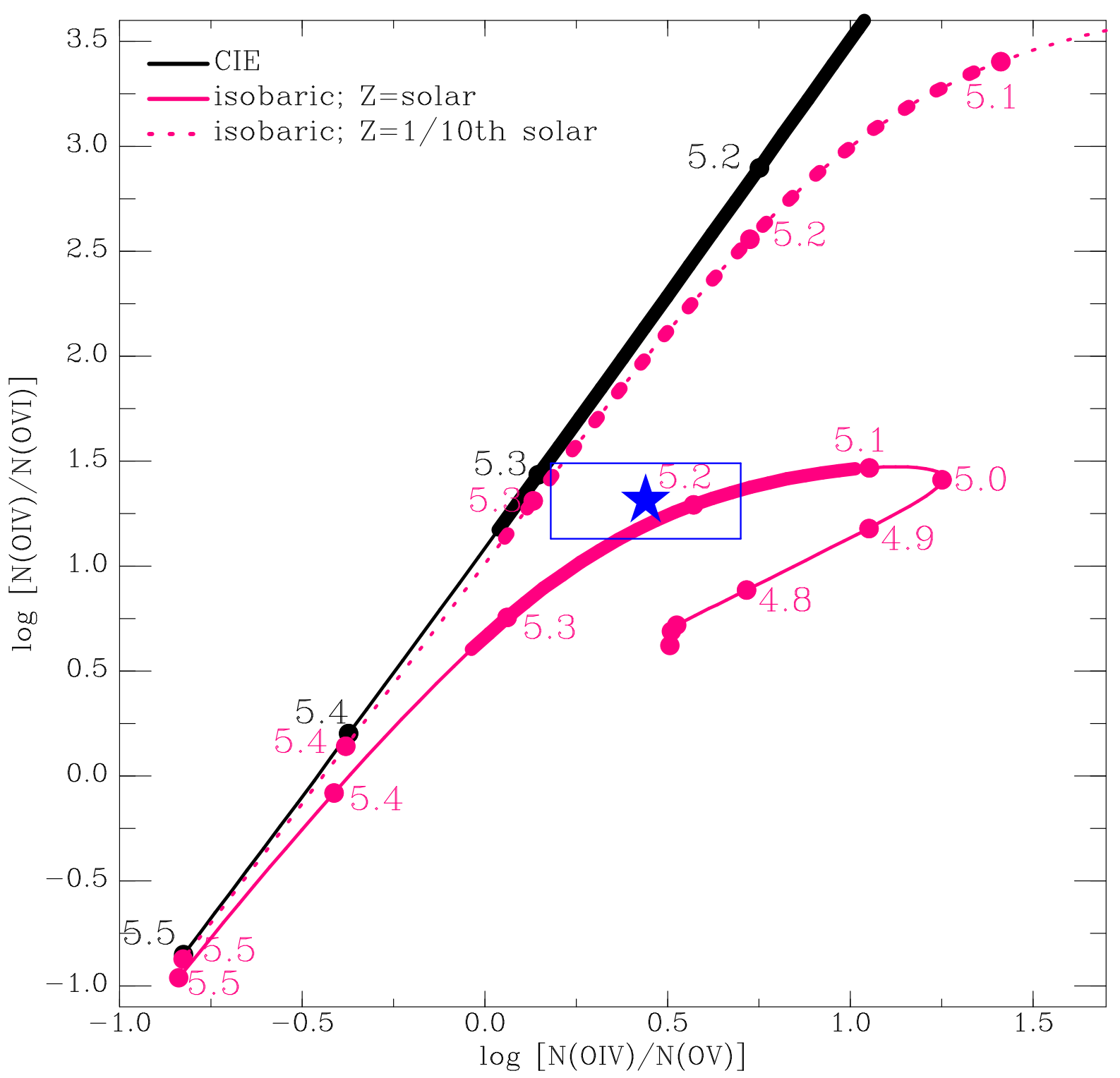

Fig. 19. Column density ratio O IV/O v vs. O IV/O vi based on the models of Gnat \& Sternberg (2007). The black line corresponds to CIE, while the red lines indicate the isobaric non-equilibrium cooling models using solar metallicity (solid line) and $0.1 \times$ solar metallicity (dotted line). Corresponding $\log T(\mathrm{~K})$ values are given by the labels along the curves. The curves are drawn as a thick line for the $\log T(\mathrm{~K})$ values corresponding to the $1 \sigma$ confidence range obtained from the RGS1 SPEX CIE fit to the OIV/O V line ratio data, extended with the systematic offset of 0.05 dex between the different CIE implementations in Gnat \& Sternberg (2007) and SPEX. The blue star and box indicate the measurement.

the cooling becomes isochoric, i.e. the density stops growing and the pressure starts to decrease as the rate of cooling increases faster than the cloud can contract (see Gnat \& Sternberg 2007 for a detailed description). Using our CIE-derived ${ }^{5}$ temperature estimate for the TTG (see Sect. 9), the estimated $N(\mathrm{H})$ is too small for the TTG to be in the isochoric phase. Thus, in the following we only consider the CIE and isobaric phases.

The independent implementations of the CIE in Gnat \& Sternberg (2007) and SPEX (Kaastra et al. 1996) may yield significant differences in the model predictions. In particular, in the SPEX package there is no option to use the solar abundance table of Asplund et al. (2009), which is implemented into the software based on Gnat \& Sternberg (2007). Instead, we use the

\footnotetext{
5 While it is in principle inconsistent to use $T$ and $N(\mathrm{H})$ obtained under the CIE assumption to investigate the boundary between the isobaric and isochoric phases, we show below that the deviation from the CIE is sufficiently small to produce a significant bias.
}

abundance table of Lodders et al. (2009) with SPEX. The cooling rate for a gas cloud with a given temperature and $N(\mathrm{H})$ is different when the abundances of the important elements $(\mathrm{O}, \mathrm{C}$, and $\mathrm{Ne}$ ) are different. In fact, for a given $N(\mathrm{O}$ IV $) / N(\mathrm{O} v)$ ratio in our measured range of [0.0, 0.7], the CIE-derived $\log T$ of Gnat \& Sternberg (2007) is about 0.05 dex higher than that in SPEX (see Fig. 19). This is comparable to the statistical uncertainties of our CIE temperature measurement. Thus, we consider this difference as an systematic uncertainty in the following.

The calculations indicated that the CIE model and the isobaric model with abundance of 0.1 solar agreed only very marginally with the measured column density ratios of O IV, O v, and O VI (see Fig. 19). On the other hand, the isobaric model with a solar abundance yields an excellent fit to the data with $\log T(\mathrm{~K}) \approx 5.2$. Our results support the Galactic Fountain model, which predicts a solar abundance for the TTG and that the gas is in a cooling transition phase. 
Yet the temperature is not low enough to produce a significant difference in the $N(\mathrm{OIV}) / N(\mathrm{OV})$ ratio for a given temperature in the CIE and non-CIE models. Thus, there is no significant bias when deriving the TTG temperature via the X-ray spectral modelling assuming CIE (see next section).

\section{Local hot bubble}

Before modelling the Galactic absorption with separate components for the TTG and the hot halo, we checked whether the local hot bubble (LHB) contributes significantly to our signal. The LHB surrounds the Sun with an extent of $\sim 100 \mathrm{pc}$ (Tanaka \& Bleeker 1977; Sanders et al. 1977; Snowden et al. 1990) and a spatially constant temperature of $k T=0.10 \pm$ $0.01 \mathrm{keV}$ (Liu et al., in prep.).

Using the data from DXL mission (Galeazzi et al. 2011), Liu et al. (in prep.) removed the Solar Wind Charge Exchange (SWCX) emission from the RASS maps in the R12 band (0.1$0.3 \mathrm{keV})$. Since the LHB dominates the emission in this band because the hot Galactic halo is too hot, these maps represent the clean LHB emission. These maps indicate that in the PKS direction the LHB emission measure is

$\mathrm{EM}_{\mathrm{LHB}} \equiv n_{e} \times n_{p} \times l_{\mathrm{LHB}}=2.6 \times 10^{-3} \mathrm{~cm}^{-6} \mathrm{pc}$,

where $l_{\mathrm{LHB}}$ is the line-of-sight path length through LHB.

Since the sound crossing time in the LHB medium $\left(\sim 10^{6} \mathrm{yrs}\right)$ is much smaller than the age of the LHB $\left(\sim 10^{7} \mathrm{yrs}\right)$, the LHB density is generally assumed to be spatially constant, measured as $n_{e}=4.7 \pm 0.5 \times 10^{-3} \mathrm{~cm}^{-3}$ by Snowden et al. (2014). Assuming the He to $\mathrm{H}$ ratio of 0.1 and complete ionisation for both $\mathrm{H}$ and $\mathrm{He}$, i.e. that $n_{e}=1.2 \times n_{p}$, we can thus estimate the total hydrogen column density of the LHB as

$$
\begin{aligned}
N(\mathrm{H}, \mathrm{LHB}) & =n_{e} \times l_{\mathrm{LHB}}=1.2 \times \frac{\mathrm{EM}_{\mathrm{LHB}}}{n_{e}} \\
& =1.2 \times \frac{2.6 \times 10^{-3} \mathrm{~cm}^{-6} \mathrm{pc}}{4.7 \times 10^{-3} \mathrm{~cm}^{-3}} \approx 2.0 \times 10^{18} \mathrm{~cm}^{-2} .
\end{aligned}
$$

This is less than $10 \%$ of the equivalent hydrogen column density of the hot halo absorber we derived in this work (see below). We estimated the effect of this component by an additional CIE absorber, with a hydrogen column density fixed to the above value and the temperature to $0.1 \mathrm{keV}$. There was a negligible change in the best-fit parameters of the hot halo and transition temperature gas. Thus, we do not include the LHB component in the following analysis.

\section{Galactic CIE absorption modelling}

Since photoionisation can be excluded as the origin of the O IV and $\mathrm{OV}$ absorption (Sect. 6.1), the deviation from equilibrium does not introduce significant bias (Sect. 7), and the LHB does not contaminate the signal significantly, we proceed to assume that these ions reside in a gas in collisionally ionised equilibrium.

\subsection{Method}

In practice, we adopted the hot model of SPEX, which calculates the transmission of a plasma with a given temperature $(k T)$, equivalent hydrogen column density $(N(\mathrm{H}))$, metal abundance $(Z)$, metal-to-H ratio, and non-thermal velocity $\left(v_{\mathrm{nt}}\right)$, assuming CIE as a function of the wavelength. Multiplied with a given background emission spectrum, the model thus produces a prediction for the absorption lines. We found that the absorption due to the intervening Local Hot Bubble in this direction is negligible compared to the $\mathrm{HH}$ component (see Sect. 8). We thus employed two hot components, one corresponding to the hot upper Galactic halo $(\mathrm{HH})$ and one to the transition temperature gas (TTG), to fit the full 9-36 ̊ band spectra.

We adopted the element number density ratios from Lodders et al. (2009). We used the above discussed range of 15$35 \mathrm{~km} \mathrm{~s}^{-1}$ (see Sect. 3) as a prior to the non-thermal velocities of each absorbing component, independently from each other.

For the LETG, we additionally included the neutral Galactic disk component (ND), which was used to correct for the cold Galactic absorption in the RGS data (see Sect. 2.1). We attempted the modelling of the blazar emission continuum in the LETG data with variants of the power-law model, but did not find a well-fitting solution. We found that a spline model with eight terms described the full band continuum acceptably. For a given individual line, the equivalent hydrogen column density and the metal abundance are fully anti-correlated. Our data were not adequate to break this degeneracy and thus we further assumed in our fits that the metal abundances are solar.

In summary, our essential choices for the analysis were

1) The collisional ionisation equilibrium is valid (as indicated by the CLOUDY analysis).

2) The abundances of the metals in HH and TTG are solar.

3) The non-thermal velocity dispersion $\sigma_{V}$ is in the range 15$35 \mathrm{~km} \mathrm{~s}^{-1}$ (as indicated by the FUV analysis in Sect. 3).

Thus, our free parameters in the fits are the temperature $k T$ and the equivalent hydrogen column density $N(\mathrm{H})$ of the two components and the continuum normalisation. For LETG, the shape of the continuum is also allowed to vary. The comparison of the above parameters obtained via RGS2 with those obtained with the other instruments is complicated. Namely, RGS2 does not cover the wavelengths of the OIV and OV lines and thus cannot constrain the TTG component. Also, RGS2 does not cover the $\mathrm{O}$ VII $1 \mathrm{~s}-2 \mathrm{p}$ line. As a result, the parameters derived with RGS2 had uncertainties that were too large to be useful and there were discrepencies with those derived with RGS1 and HRC. Thus, we do not discuss the RGS2 measurements further for the CIE analysis. Given the problems of the systematically inconsistent line measurements with LETG/ACIS-S (see Sect. 4.1.3), in particular the non-detection of the O IV and OV lines of the TTG component, we also excluded the LETG/ACIS-S data from the further analysis.

Considering a single line with a temperature near the value of the peak strength, higher column densities can to an extent compensate for lower ionisation factors when reproducing the given line data. Thus, we expect some level of correlation between the temperature and the equivalent hydrogen column density in the spectral analysis. In order to consider this correlation (and possible correlations between the different absorbing components) we adopted the following procedure to estimate the best-fit parameters and their uncertainties for each absorber (HH and TTG), and for the consequent O IV and O v column densities for TTG. The procedure was applied independently to the RGS1 and HRC data as follows:

1. We obtained the global best-fit $N(\mathrm{H})-k T$ combination by fitting the full band data with the two-temperature CIE absorber model (plus a fixed neutral absorber for HRC) using a $\chi^{2}$ minimisation, thus yielding $\chi_{\text {min,global }}^{2}$ to be used below. 
2. We then determined dense $2 \mathrm{D}$ grids around the best-fit values in the $N(\mathrm{H})-k T$ parameter space for both $\mathrm{HH}$ and TTG.

3. We fixed the $N(\mathrm{H})-k T$ value pair of a given component to one defined by the above grid.

4. We re-fitted the data with $N(\mathrm{H})-k T$ of the other component, as well as the normalisation of the continuum as free parameters, thus obtaining $\chi_{\min , \mathrm{i}}^{2}$.

5. We recorded the difference $\Delta \chi^{2}$ between the $\chi^{2}$ values of the above best-fit $\left(\chi_{\min , \mathrm{i}}^{2}\right)$ and the global best-fit $\left(\chi_{\min , \text { global }}^{2}\right)$.

6. We repeated steps $2-5$, but this time we chose a different combination of $N(\mathrm{H})-k T$ value pair.

7. We repeated steps $2-6$ but this time for the other absorber.

8. When all the value combinations in the predefined grid had been used, we constructed 2D $\Delta \chi^{2}$ maps corresponding to our $N(\mathrm{H})-k T$ grids for $\mathrm{HH}$ and TTG.

9. We determined the $1 \sigma$ subspace of the grid by selecting only such value combinations, which yielded $\Delta \chi^{2} \leq 2.3$.

10. We used the maximal variation of the projection of the allowed parameter subspace into $N(\mathrm{H})$ and $k T$ axes as $1 \sigma$ uncertainty intervals.

11. Using each best fit with the $N(\mathrm{H})-k T$ value combinations within the allowed $1 \sigma$ subspace for TTG, we computed the distribution of the allowed O IV and OV column densities and adopted the extremal values as the $1 \sigma \mathrm{CL}$ interval.

\subsection{Results}

The best-fit multicomponent CIE models (see Table 5) described the data reasonably well (see Fig. 20 for the RGS1 fits). However, the C VI and N VI lines at the longest wavelengths were not well modelled. This may indicate the existence of an additional component of the hot halo with a slightly lower temperature than reported above. We will investigate this issue further in a future work.

\subsubsection{TTG}

Using only the statistical uncertainties the RGS1 data constrained the TTG parameters as $N\left(\mathrm{H}_{\mathrm{TTG}}\right)=1.0[0.6-1.3] \times$ $10^{19} \frac{Z_{\odot}}{Z_{\mathrm{TTG}}} \mathrm{cm}^{-2}$ and $k T_{\mathrm{TTG}}=1.4[1.2-1.5] \times 10^{-2} \mathrm{keV}$ $\left(\log T_{\mathrm{TTG}}(\mathrm{K})=5.2[5.1-5.3]\right)$. These are in excellent agreement with the independent HRC measurements $N\left(\mathrm{H}_{\mathrm{TTG}}\right)=$ $0.9[0.5-1.5] \times 10^{19} \frac{Z_{\odot}}{Z_{\mathrm{TTG}}} \mathrm{cm}^{-2}$ and $k T_{\mathrm{TTG}}=1.5[1.3-1.8] \times$ $10^{-2} \mathrm{keV}\left(\log T_{\text {TTG }}(\mathrm{K})=5.2[5.1-5.3]\right.$; see Fig. 21$)$.

At the above temperature O IV is the dominating oxygen ion. The O IV column density distributions are smooth, but in case of RGS1 this distribution peaks at a slightly lower value than that corresponding to the global best-fit model (see Sect. 9.1). We thus adopted the peak location as the most likely value for RGS1, obtaining consistent values $N(\mathrm{OIV}, \mathrm{RGS} 1)=3.6[2.4-5.1] \times$ $10^{15} \mathrm{~cm}^{-2}$ (see Fig. 21) and $N(\mathrm{OIV}, \mathrm{HRC})=3.3[1.6-5.3] \times$ $10^{15} \mathrm{~cm}^{-2}$.

The above consistencies imply that the uncertainties of the calibration of the effective areas of RGS1 and HRC (see Sect. 2.1) do not introduce a significant bias to our results of the CIE analysis of the TTG. However, we must estimate the effect of the calibration uncertainties to the error bars of the parameters derived above. Since we do not know the probability distributions of the calibration uncertainties of both the RGS 1 and HRC, we cannot perform a proper error propagation analysis. Rather, we approximated the effect by adding $2 \%$ of the best-fit model flux to the statistical uncertainties at each spectral bin and
Table 5. Results of multi-temperature CIE modelling.

\begin{tabular}{lcccc}
\hline \hline & $\begin{array}{c}N_{\mathrm{H}} \\
\left(10^{19} \frac{Z_{\odot}}{Z_{\text {TTG }}} \mathrm{cm}^{-2}\right)\end{array}$ & $\begin{array}{c}N_{\text {O IV }} \\
\left(10^{15} \mathrm{~cm}^{-2}\right)\end{array}$ & $\begin{array}{c}k T \\
(\mathrm{keV})\end{array}$ & $\log T(\mathrm{~K})$ \\
\hline \multicolumn{5}{c}{ RGS1 } \\
TTG & $1.0 \pm 0.5$ & $3.6 \pm 2.0$ & $1.4 \pm 0.3 \times 10^{-2}$ & $5.2 \pm 0.1$ \\
HH & $2.7 \pm 0.8$ & - & $1.6 \pm 0.2 \times 10^{-1}$ & $6.3 \pm 0.1$ \\
\multicolumn{5}{c}{ HRC } \\
TTG & $0.9 \pm 0.5$ & $3.3 \pm 2.0$ & $1.5 \pm 0.3 \times 10^{-2}$ & $5.2 \pm 0.1$ \\
HH & $3.1 \pm 1.2$ & - & $1.6 \pm 0.3 \times 10^{-1}$ & $6.3 \pm 0.1$ \\
\hline
\end{tabular}

Notes. Including systematic $2 \%$ uncertainties.

we repeated the above analysis (see Sect. 9.1). This had a significant effect on RGS1; the $1 \sigma$ uncertainty intervals increased from [0.6-1.3] to [0.5-1.4] $\times 10^{19} \frac{Z_{\odot}}{Z_{\mathrm{TTG}}} \mathrm{cm}^{-2}$ and [1.2-1.5] to [0.9-1.6] $\times 10^{-2} \mathrm{keV}$ for $N\left(\mathrm{H}_{\mathrm{TTG}}\right)$ and $k T_{\mathrm{TTG}}$, respectively.

The inclusion of the systematic uncertainties rendered the RGS1 OV column density rather uncertain. However, we obtained useful constraints for the RGS1 O IV column density, i.e. $N(\mathrm{OIV}, \mathrm{RGS} 1)=3.6[1.9-5.8] \times 10^{15} \mathrm{~cm}^{-2}$ (see Fig. 21$)$, which is consistent with the HRC value of $N(\mathrm{OIV}, \mathrm{HRC})=$ $3.3[1.5-5.5] \times 10^{15} \mathrm{~cm}^{-2}$. The values are consistent with those derived without an assumption about the ionisation balance (see Sect. 5 and Table 3). In addition to the results of the CLOUDY analysis (see Sect. 6.1) this indicates that the photoionisation is not significantly contaminating the TTG absorption signal.

The larger statistical uncertainties of the HRC data render the calibration uncertainty effect negligible on the results derived with the HRC. Because of the larger statistical HRC uncertainties and the significant level of systematic uncertainties in the RGS1 data, the weighted averages of RGS1 and HRC values are less precise than the results obtained with RGS1 alone, using $2 \%$ systematic uncertainties and symmetrising the confidence interval, i.e.

$$
\begin{aligned}
& N\left(\mathrm{H}_{\mathrm{TTG}}\right)=1.0 \pm 0.5 \times 10^{19} \frac{Z_{\odot}}{Z_{\mathrm{TTG}}} \mathrm{cm}^{-2} \\
& k T_{\mathrm{TTG}}=1.4 \pm 0.3 \times 10^{-2} \mathrm{keV} \\
& \log T_{\mathrm{TTG}}(\mathrm{K})=5.2 \pm 0.1 \\
& N(\mathrm{O} \text { IV })=3.6 \pm 2.0 \times 10^{15} \mathrm{~cm}^{-2}
\end{aligned}
$$

\subsubsection{Hot halo}

We repeated the analysis of the previous section, but this time for the hot halo $(\mathrm{HH})$ component, including the $2 \%$ systematic uncertainties. The results for RGS1 and HRC are consistent (see Table 5). As in the case of TTG, the weighted averages of RGS1 and HRC measurements are less precise than those obtained with RGS1 alone, which yielded

$$
\begin{aligned}
& N\left(H_{\mathrm{HH}}\right)=2.7 \pm 0.8 \times 10^{19} \frac{Z_{\odot}}{Z_{\mathrm{TTG}}} \mathrm{cm}^{-2} \\
& k T_{\mathrm{HH}}=1.6 \pm 0.2 \times 10^{-1} \mathrm{keV} \\
& \log T_{\mathrm{HH}}(\mathrm{K})=6.3 \pm 0.1 .
\end{aligned}
$$

\section{Conclusions}

We analysed $\sim 3$ Ms of data of PKS 2155-304 obtained with high-resolution X-ray spectrometers RGS1 and RGS2 on board 


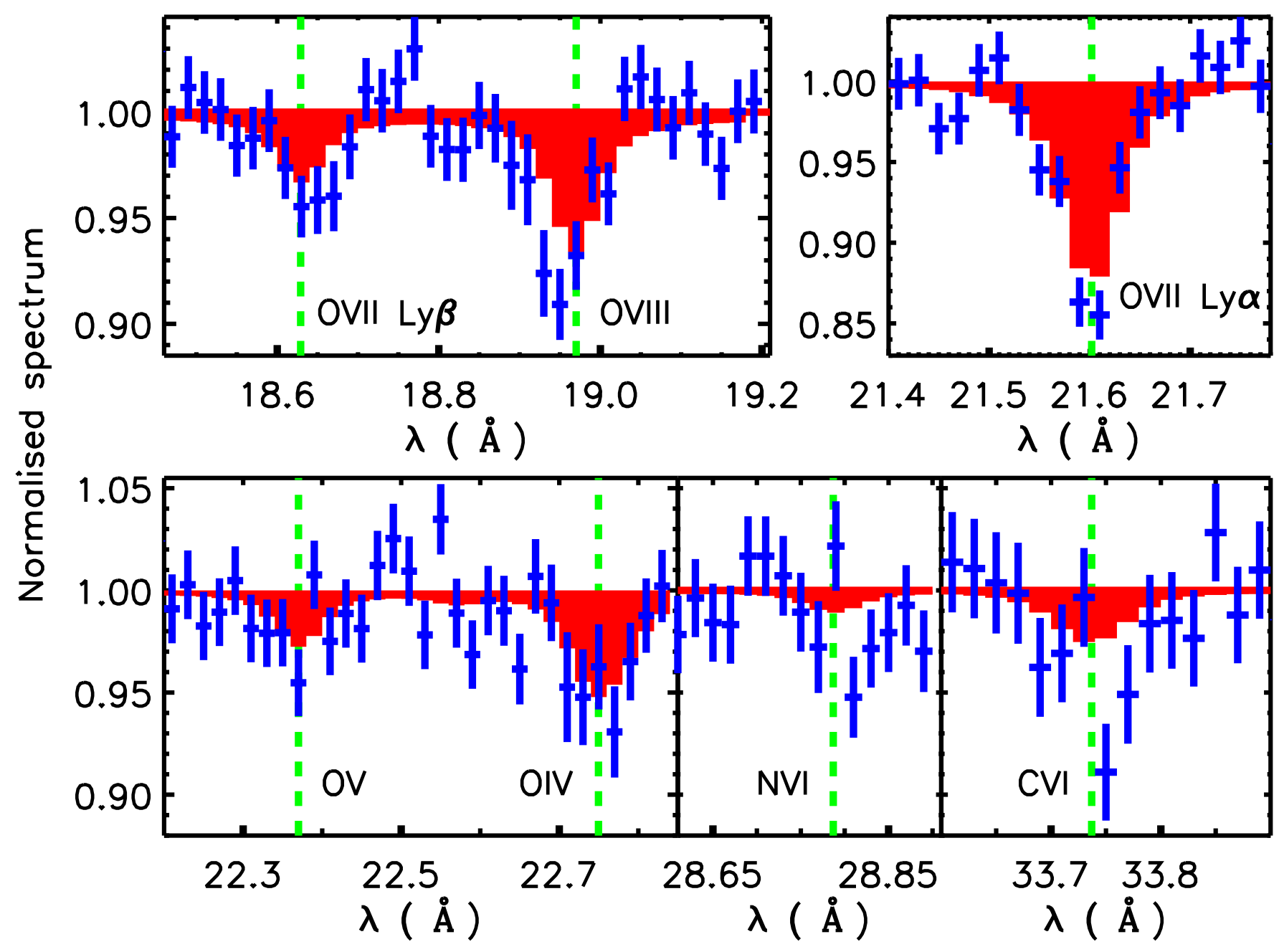

Fig. 20. Best-fit CIE model (red lines) around the lines significantly detected with RGS1 data (blue crosses).

XMM-Newton and LETG/HRC-S and LETG/ACIS-S on board Chandra. The main conclusions are listed below.

- Due to the high statistical quality of the data and the coverage of the important wavelengths with more than one instrument, we obtained secure detection of 10 Galactic absorption lines at CL higher than $99.75 \%$ in the sight line towards PKS 2155-304. Owing to our very strict criteria for line detection, we may have omitted some important lines at problematic wavelengths.

- We discovered a significant absorption blend from inner transitions of O IV, i.e. $1 \mathrm{~s}-2 \mathrm{p}^{2} \mathrm{~S}(22.571 \AA), 1 \mathrm{~s}-2 \mathrm{p}^{2} \mathrm{P}(22.741 \AA)$ and $1 \mathrm{~s}-2 \mathrm{p}{ }^{2} \mathrm{D}(22.777 \AA)$, and from O v 1s-2p (22.370 $)$, consistent with RGS1 and LETG/HRC-S.

- Combining our X-ray measurements of PKS 2155-304 with those obtained in FUV we determined that the OIV and $\mathrm{O} \mathrm{V}$ absorption cannot be produced by photoionisation, since the observed column densities would imply an ionisation parameter that would overpredict the FUV-observed C IV column density by an order of magnitude.

- Using non-CIE cooling models of Gnat \& Sternberg (2007), we showed that the column density ratios of O IV, O V, and $\mathrm{O}$ VI are in excellent agreement with a model where all these ions originate in isobarically cooling gas with solar abundances and a temperature of $\log T(\mathrm{~K}) \sim 5.2$. The CIE model and an isobaric 0.1 solar model are strongly disfavoured by the data. We thus identify this absorber with the transition temperature gas TTG as predicted by the Galactic fountain theory, previously detected only in FUV (e.g. Wakker et al. 2012).

- The temperature derived from the non-CIE modelling is not low enough to produce a significant difference between the temperature based on the $\mathrm{O} \mathrm{IV} / \mathrm{O} \mathrm{V}$ column density ratio assuming CIE or non-CIE.

- Assuming CIE, the spectral fit to the PKS 2155-304 spectrum, driven by the $\mathrm{O} I \mathrm{~V} / \mathrm{O} \mathrm{V}$ ratio, yielded a temperature of $k T=1.4 \pm 0.3 \times 10^{-2} \mathrm{keV}$, i.e. $\log T(\mathrm{~K})=5.2 \pm 0.1$ for TTG.

- The column density of O IV, obtained with CIE assumption $\left(N_{\text {OIV }}=3.6[1.9-5.8] \times 10^{15} \mathrm{~cm}^{-2}\right)$ agrees with that derived directly from the EW measurement of the line without assumptions about the phase of the gas.

- The equivalent hydrogen column density corresponding to the O IV absorber is $1.0 \pm 0.5 \times 10^{19} \frac{Z_{\odot}}{Z_{\mathrm{TTG}}} \mathrm{cm}^{-2}$.

- The significant discrepancy in the measured EW of the residual signal at the instrumental feature at $\lambda=23.35 \AA$ indicates that the signal cannot be purely due to astrophysical O II, as suggested by Nicastro et al. (2016).

Acknowledgements. J.N. is funded by PUT246 grant from Estonian Research Council. Support for BPW was provided by NASA through grants HSTGO-13892.01-A, HST-GO-13840.05-A and HST-GO-13721.02-A from the Space Telescope Science Institute, which is operated by the Association of 

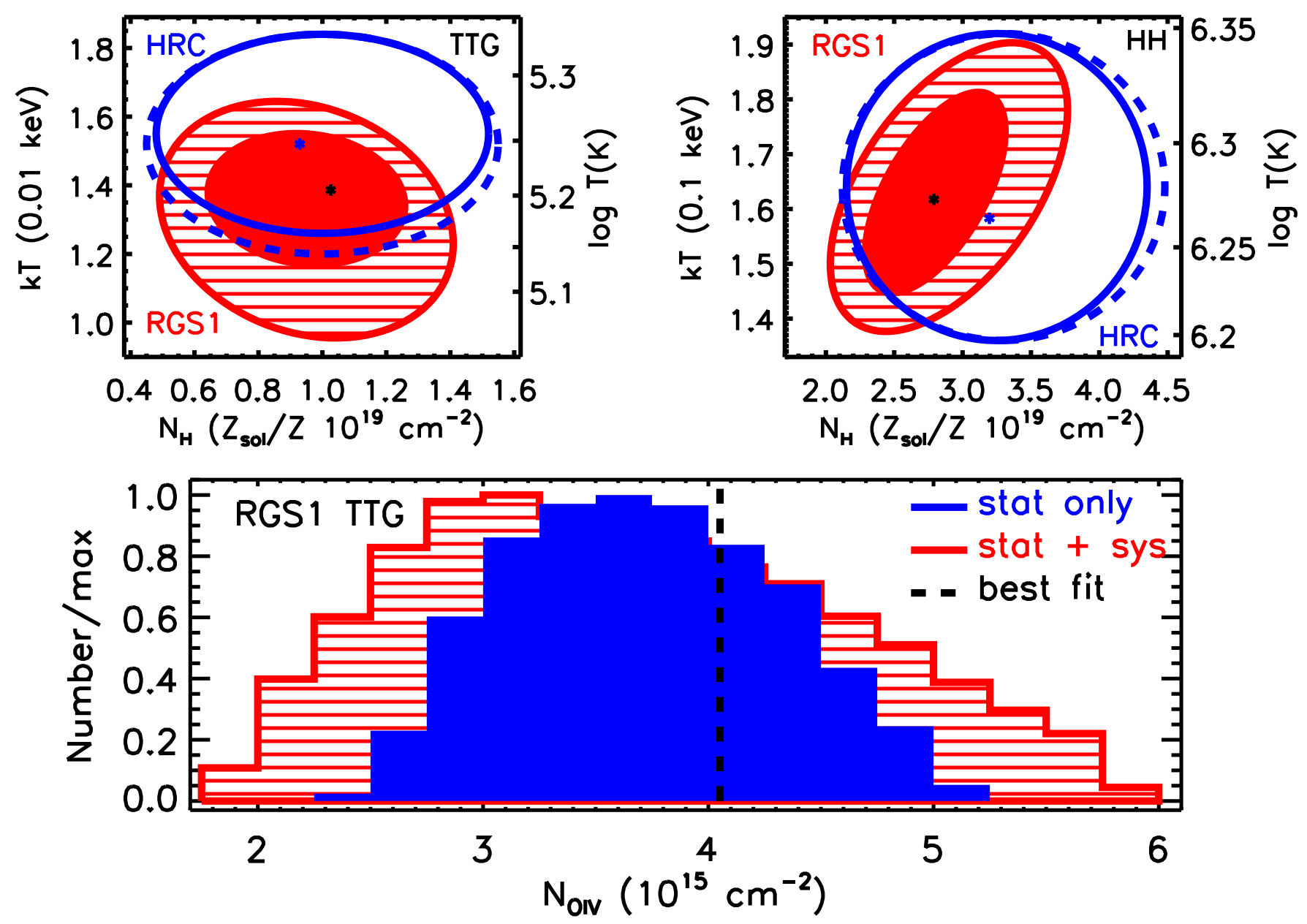

Fig. 21. Uncertainties of $1 \sigma$ in the $k T-N_{\mathrm{H}}$ plane for TTG (upper left panel) and HH (upper right panel) for RGS1 (red) and HRC(blue). The results, obtained when using only statistical uncertainties, are indicated with the filled ellipses (RGS1) and solid blue lines (HRC). The red shaded regions and the blue dashed line indicate the results obtained when including the $2 \%$ uncertainty for the effective area calibration uncertainty. The global best-fit spectral model parameters are indicated as black (RGS1) and blue (HRC) asterisks. The lower panel shows the distribution of the O IV column density of TTG, corresponding to the allowed RGS $1 k T-N_{\mathrm{H}}$ region in the upper left panel. The results obtained with only statistical uncertainties are indicated with a blue region, while the red region shows the results when including the $2 \%$ systematics. The dashed line indicates the value corresponding to the global best-fit spectral model.

Universities for Research in Astronomy. We thank J. de Plaa, J. Drake and E. Saar for help.

\section{References}

Asplund, M., Grevesse, N., Sauval, A. J., \& Scott, P. 2009, ARA\&A, 47, 481 Burrows, D. N., \& Mendenhall, J. A. 1991, Nature, 351, 629

Costantini, E., Pinto, C., Kaastra, J. S., et al. 2012, A\&A, 539, A32

de Vries, C. P., den Herder, J. W., Kaastra, J. S., et al. 2003, A\&A, 404, 959

Fang, T., Canizares, C. R., \& Yao, Y. 2007, ApJ, 670, 992

Fang, T., Buote, D., Bullock, J., \& Ma, R. 2015, ApJS, 217, 21

Ferland, G. J., Korista, K. T., Verner, D. A., et al. 1998, PASP, 110, 761

Fox, A. J., Wakker, B. P., Savage, B. D., et al. 2005, ApJ, 630, 332

Galeazzi, M., Chiao, M., Collier, M. R., et al. 2011, Exp. Astron., 32, 83

Gnat, O., \& Sternberg, A. 2007, ApJS, 168, 213

Gu, M. F., Schmidt, M., Beiersdorfer, P., et al. 2005, ApJ, 627, 1066

Haardt, F., \& Madau, P. 2001, in Clusters of Galaxies and the High Redshift Universe Observed in X-rays, eds. D. M. Neumann, \& J. T. V. Tran

Hagihara, T., Yao, Y., Yamasaki, N. Y., et al. 2010, PASJ, 62, 723

Kaastra, J. S. 2017, Astron. Nachr., 338, 146

Kaastra, J. S., Mewe, R., \& Nieuwenhuijzen, H. 1996, in UV and X-ray Spectroscopy of Astrophysical and Laboratory Plasmas, eds. K. Yamashita, \& T. Watanabe, 411

Kaastra, J. S., Steenbrugge, K. C., Raassen, A. J. J., et al. 2002, A\&A, 386, 427

Kaastra, J. S., Paerels, F. B. S., Durret, F., Schindler, S., \& Richter, P. 2008, Space Sci. Rev., 134, 155

Kaastra, J. S., de Vries, C. P., Steenbrugge, K. C., et al. 2011, A\&A, 534, A37
Kaastra, J., de Vries, C., \& den Herder, J. 2015, Effective area calibration of the RGS, SRON internal report

Lodders, K., Palme, H., \& Gail, H.-P. 2009, Landolt Börnstein [arXiv:0901.1149]

Nicastro, F., Senatore, F., Gupta, A., et al. 2016, MNRAS, 457, 676

Pinto, C., Kaastra, J. S., Costantini, E., \& de Vries, C. 2013, A\&A, 551, A25

Rasmussen, A. P., Kahn, S. M., Paerels, F., et al. 2007, ApJ, 656, 129

Sakai, K., Yao, Y., Mitsuda, K., et al. 2014, PASJ, 66, 83

Sanders, W. T., Kraushaar, W. L., Nousek, J. A., \& Fried, P. M. 1977, ApJ, 217, L87

Savage, B. D., \& Wakker, B. P. 2009, ApJ, 702, 1472

Sembach, K. R., Savage, B. D., Lu, L., \& Murphy, E. M. 1999, ApJ, 515, 108

Shapiro, P. R., \& Field, G. B. 1976, ApJ, 205, 762

Shull, J. M., Danforth, C. W., \& Tilton, E. M. 2014, ApJ, 796, 49

Snowden, S. L., Cox, D. P., McCammon, D., \& Sanders, W. T. 1990, ApJ, 354, 211

Snowden, S. L., Mebold, U., Hirth, W., Herbstmeier, U., \& Schmitt, J. H. M. 1991, Science, 252, 1529

Snowden, S. L., Chiao, M., Collier, M. R., et al. 2014, ApJ, 791, L14

Tanaka, Y., \& Bleeker, J. A. M. 1977, Space Sci. Rev., 20, 815

Verner, D. A., Ferland, G. J., Korista, K. T., \& Yakovlev, D. G. 1996, ApJ, 465, 487

Wakker, B. P., Savage, B. D., Sembach, K. R., et al. 2003, ApJS, 146, 1

Wakker, B. P., Lockman, F. J., \& Brown, J. M. 2011, ApJ, 728, 159

Wakker, B. P., Savage, B. D., Fox, A. J., Benjamin, R. A., \& Shapiro, P. R. 2012, ApJ, 749, 157

Williams, R. J., Mathur, S., Nicastro, F., \& Elvis, M. 2007, ApJ, 665, 247 


\section{Appendix A: Observation log}

We provide here information about the XMM-Newton and Chandra observations used in this work.

Table A.1. RGS observations.

\begin{tabular}{ccccc}
\hline \hline Orbit & obsid & norm $^{a}$ & $\Gamma^{b}$ & $t^{c}(\mathrm{ks})$ \\
\hline 174 & 0080940101 & 4069 & 2.69 & 59 \\
174 & 0080940301 & 3288 & 2.76 & 60 \\
362 & 0124930301 & 8558 & 2.65 & 90 \\
450 & 0124930501 & 3782 & 2.55 & 102 \\
545 & 0124930601 & 2867 & 2.59 & 114 \\
724 & 0158960101 & 2120 & 2.85 & 27 \\
908 & 0158960901 & 2397 & 2.87 & 29 \\
908 & 0158961001 & 3217 & 2.77 & 40 \\
993 & 0158961101 & 4442 & 2.64 & 29 \\
1095 & 0158961301 & 4714 & 2.71 & 60 \\
1171 & 0158961401 & 1836 & 2.69 & 65 \\
1266 & 0411780101 & 2682 & 2.64 & 100 \\
1349 & 0411780201 & 4613 & 2.77 & 68 \\
1543 & 0411780301 & 5463 & 2.68 & 61 \\
1734 & 0411780401 & 3578 & 2.87 & 65 \\
1902 & 0411780501 & 1843 & 2.79 & 71 \\
2084 & 0411780601 & 3053 & 2.56 & 64 \\
2268 & 0411780701 & 708 & 2.85 & 57 \\
2449 & 0411782101 & 1579 & 2.74 & 76 \\
2542 & 0727770101 & 1724 & 2.60 & 95 \\
2631 & 0727770501 & 1900 & 2.76 & 88 \\
2633 & 0727770901 & 1676 & 2.82 & 64 \\
2632 & 0727771001 & 1498 & 2.74 & 39 \\
2632 & 0727771101 & 1879 & 2.83 & 39 \\
2726 & 0727771301 & 1336 & 2.64 & 92 \\
& & & & \\
& & & \\
& & & &
\end{tabular}

Notes. ${ }^{(a)}$ The normalisation of the absorbed power-law fitted to the RGS data over the full band; the units are $4 \pi \times$ the number of photons $\mathrm{m}^{-2} \mathrm{~s}^{-1} \mathrm{keV}^{-1}$ at $1 \mathrm{keV}$. (b) The best-fit photon index of the power-law described above. ${ }^{(c)}$ The exposure time.
Table A.2. LETG observations.

\begin{tabular}{|c|c|c|}
\hline OBS. ID. & Detector & Exposure (ks) \\
\hline 1015 & ACIS-456789 & 9.5 \\
\hline 10662 & ACIS-456789 & 28.4 \\
\hline 11965 & ACIS-456789 & 28.4 \\
\hline 13096 & ACIS-456789 & 26.5 \\
\hline 14265 & ACIS-456789 & 26.5 \\
\hline 15475 & ACIS-456789 & 28.4 \\
\hline 16423 & ACIS-56789 & 28.1 \\
\hline 1703 & ACIS-456789 & 25.3 \\
\hline 1790 & ACIS-456789 & 20.9 \\
\hline 1791 & ACIS-456789 & 20.9 \\
\hline 1792 & ACIS-456789 & 20.9 \\
\hline 1793 & ACIS-456789 & 20.9 \\
\hline 1794 & ACIS-456789 & 20.9 \\
\hline 1795 & ACIS-456789 & 19.7 \\
\hline 1796 & ACIS-456789 & 19.5 \\
\hline 1797 & ACIS-456789 & 19.5 \\
\hline 1798 & ACIS-456789 & 19.5 \\
\hline 1799 & ACIS-456789 & 19.9 \\
\hline 2323 & ACIS-456789 & 9.0 \\
\hline 2324 & ACIS-456789 & 8.6 \\
\hline 2335 & ACIS-456789 & 29.1 \\
\hline 3168 & ACIS-456789 & 28.8 \\
\hline 3667 & ACIS-456789 & 14.2 \\
\hline 3668 & ACIS-456789 & 13.5 \\
\hline 3669 & ACIS-456789 & 42.4 \\
\hline 3707 & ACIS-456789 & 26.9 \\
\hline 4416 & ACIS-456789 & 46.5 \\
\hline 6090 & ACIS-456789 & 27.5 \\
\hline 6091 & ACIS-456789 & 29.2 \\
\hline 6874 & ACIS-456789 & 28.5 \\
\hline 6924 & ACIS-456789 & 9.5 \\
\hline 6927 & ACIS-456789 & 27.0 \\
\hline 7293 & ACIS-456789 & 8.5 \\
\hline 8388 & ACIS-456789 & 29.3 \\
\hline 9704 & ACIS-456789 & 27.8 \\
\hline 9706 & ACIS-456789 & 9.5 \\
\hline 9708 & ACIS-456789 & 9.2 \\
\hline 9710 & ACIS-456789 & 9.2 \\
\hline \multirow[t]{2}{*}{9713} & ACIS-456789 & 29.7 \\
\hline & Total & 900 \\
\hline 1013 & HRC-S & 26.6 \\
\hline 3709 & HRC-S & 13.7 \\
\hline 7294 & HRC-S & 10.0 \\
\hline 1704 & HRC-S & 25.9 \\
\hline 7295 & HRC-S & 10.1 \\
\hline 4406 & HRC-S & 13.8 \\
\hline 8379 & HRC-S & 29.8 \\
\hline 5172 & HRC-S & 26.9 \\
\hline 9707 & HRC-S & 10.1 \\
\hline 3166 & HRC-S & 29.8 \\
\hline 6922 & HRC-S & 10.0 \\
\hline 9709 & HRC-S & 10.1 \\
\hline 331 & HRC-S & 62.7 \\
\hline \multirow[t]{2}{*}{6923} & HRC-S & 29.9 \\
\hline & Total & 300 \\
\hline
\end{tabular}

